\title{
À propos des conséquences du réchauffement climatique dans les régions où existe un pergélisol Albert Pissart
}

\section{Résumé}

Les régions arctiques et de montagne où le sol est gelé toute l'année contiennent souvent des masses de glace importantes. Un réchauffement climatique en provoquant leur fusion entraînera des modifications considérables dans ces régions et détruira de nombreuses installations humaines. C'est l'état des connaissances concernant cette importante question qui est présenté cidessous, en montrant qu'en raison du rôle complexe de nombreux facteurs qui déterminent la température au sommet du pergélisol, des prévisions à court terme ne peuvent être avancées aujourd'hui.

\section{Citer ce document / Cite this document :}

Pissart Albert. À propos des conséquences du réchauffement climatique dans les régions où existe un pergélisol. In: Bulletin de la Classe des sciences, tome $5, n^{\circ} 7-12,1994$. pp. 279-308;

doi : https://doi.org/10.3406/barb.1994.27559

https://www.persee.fr/doc/barb_0001-4141_1994_num_5_7_27559

Fichier pdf généré le 05/06/2020 


\title{
EXPOSÉ
}

\section{À propos des conséquences du réchauffement climatique dans les régions où existe un pergélisol}

\author{
par Albert Pissart \\ Correspondant de la Classe
}

\begin{abstract}
Résumé
Les régions arctiques et de montagne où le sol est gelé toute l'année contiennent souvent des masses de glace importantes. Un réchauffement climatique en provoquant leur fusion entraînera des modifications considérables dans ces régions et détruira de nombreuses installations humaines. C'est l'état des connaissances concernant cette importante question qui est présenté ci-dessous, en montrant qu'en raison du rôle complexe de nombreux facteurs qui déterminent la température au sommet du pergélisol, des prévisions à court terme ne peuvent être avancées aujourd'hui.
\end{abstract}

\section{Sommaire}

\section{A. Le pergélisol.}

B. La glace du sol.

B.1. LA GLACE DE SÉGRÉGATION.

B.1.1. Mécanisme de formation.

B.1.2. Conditions requises pour que la glace de ségrégation apparaisse.

B.2. La GLACE DE FENTE DE GEL.

B.2.1. Mécanisme de formation.

B.2.2. Les facteur's qui contrôlent la formation des fentes de gel.

B.2.3. Apparence en surface de la glace de fente de gel, les polvgones de fente de gel. 
B.3. LA GLACE D'INJECTION ET LES PINGOS.

B.3.1. Mécanisme de formation des pingos.

B.3.2. Importance de la glace d'injection.

B.4. LeS AUTRES TYPES DE GLACE DU SOL.

B.5. IMPORTANCE ET RÉPARTITION DE LA GLACE DU SOL.

C. Complexité des relations existant entre la température de l'air et la température au sommet du pergélisol.

D. Les phénomènes thermokarstiques.

E. Le réchauffement climatique des régions arctiques : les incertitudes et les difficultés d'en prévoir les conséquences.

F. Remarques finales.

\section{A. Le pergélisol : définition, extension géographique, et variation de température dans le sol}

Le pergélisol (en anglais permafrost = permanently frozen ground) est défini uniquement en considérant la température du sol et non l'état de l'eau (gelée ou liquide) qu'il contient. Un sol sous $0{ }^{\circ} \mathrm{C}$ peut, en effet, ne pas contenir d'eau (au sein d'une roche cohérente, par exemple) ou en contenir qui ne soit pas gelée (eau salée, ou eau tenue par des forces capillaires). Le terme pergélisol exprime donc la condition thermique inférieure à $0^{\circ} \mathrm{C}$, sous laquelle la croûte terrestre (roches, sols minéraux ou organiques) peut se trouver. En outre, le terme n'est utilisé que si cette température inférieure à $0{ }^{\circ} \mathrm{C}$ se maintient pendant au moins 2 ans. Comme le montre la figure 1 , les régions où existe de nos jours un pergélisol couvre une étendue importante puisqu'il s'étend sur environ $1 / 5$ des terres émergées. Il couvre environ $80 \%$ de l'Alaska, $50 \%$ du Canada où il atteint $51^{\circ}$ de latitude nord et $47,5 \%$ de l'ancienne U.R.S.S.

La figure 2 explique la signification des différents termes mentionnés dans la légende de la figure 1. Au nord, le pergélisol est continu et peut atteindre des centaines de mètres d'épaisseur. Plus au sud, il fait place au pergélisol discontinu au sein duquel on trouve des parties non gelées. Enfin à sa bordure méridionale, on trouve seulement des îlots de pergélisol dans des sites favorables : c'est le domaine du pergélisol sporadique. 


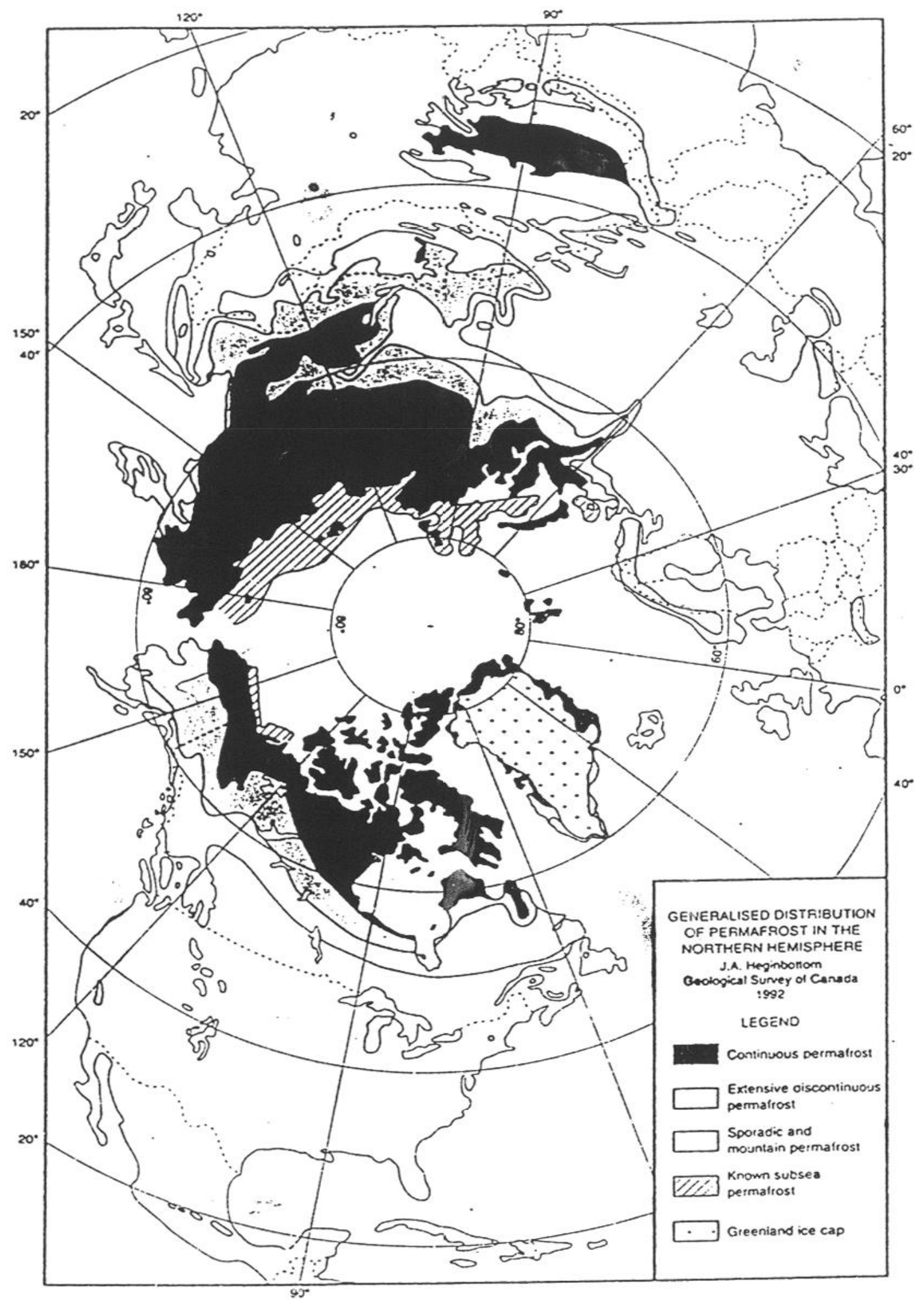

FIG. 1. - Carte du pergélisol dans l'hémisphère nord. (J. A. Heginbottom, 1993). 


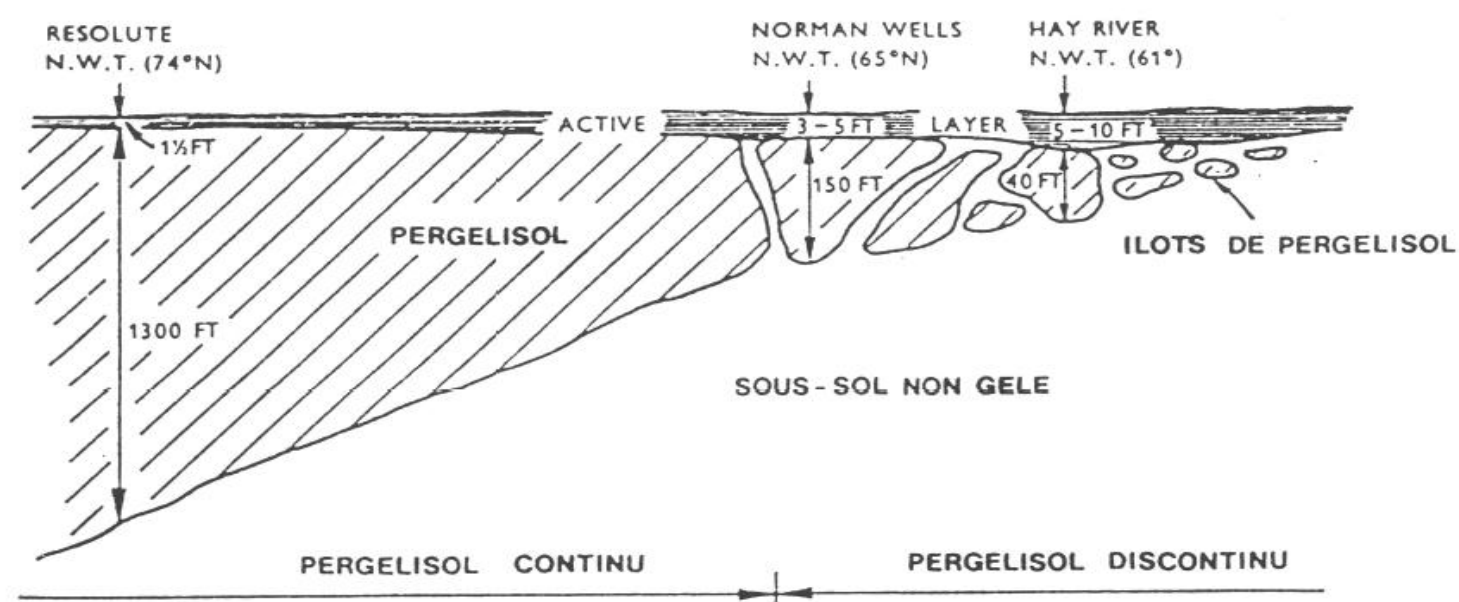

FIG. 2. - Profil N-S à travers le Canada montrant l'épaisseur variable du pergélisol et de la couche active (Brown, 1970).

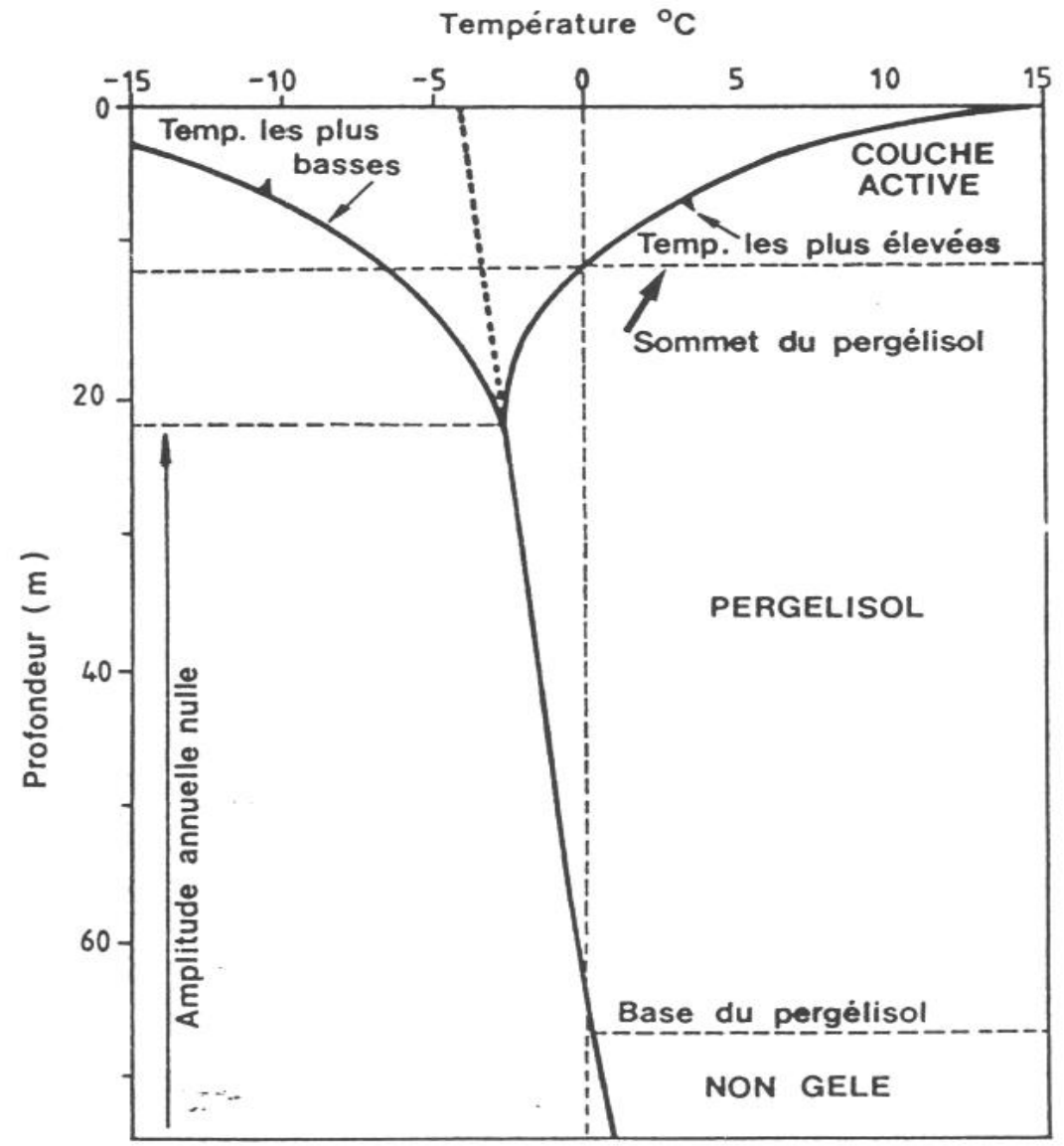

Fig. 3. - Courbes donnant l'allure des températures en profondeur et des variations annuelles extrêmes de celles-ci dans une région de pergélisol continu. Dans la couche active, les températures fluctuent de part et d'autre de $0^{\circ} \mathrm{C}$; à la partic supérieure du pergélisol, des variations existent, mais le sol reste constamment sous $0{ }^{\circ} \mathrm{C}$ (Harris, 1986). 
Au-dessus du pergélisol, s'étend la couche active en entendant par là, la couche qui gèle et dégèle chaque année. Son épaisseur augmente vers le sud qui bénéficie d'étés plus chauds et plus longs. La couche active est généralement comprise entre 0,2 et $1 \mathrm{~m}$, mais peut atteindre parfois $3 \mathrm{~m}$. Son épaisseur ne varie pas seulement avec les caractéristiques climatiques, mais aussi avec la nature du sol et principalement sa teneur en eau. Rappelons en effet à ce sujet que la chaleur latente de fusion de la glace est fort élevée : $333,54 \mathrm{~J} / \mathrm{g}$. Comme la chaleur spécifique du sol sec est de $0,8 \mathrm{~J} / \mathrm{g}$, la quantité de chaleur nécessaire pour fondre $1 \mathrm{~g}$ de glace permet d'élever de $400{ }^{\circ} \mathrm{C}, 1 \mathrm{~g}$ de sol sec. Cette observation montre combien le taux d'humidité du sol contrôle les phénomènes de gel et de dégel qui se produisent dans le sol.

Dans la couche active et à la partie supérieure du pergélisol les températures varient au cours de l'année (fig. 3). En-dessous d'une profondeur d'une vingtaine de mètres, les variations annuelles de température deviennent imperceptibles. En-dessous de cette profondeur, la température du sol s'élève progressivement en relation avec le degré géothermique. La température au sein du pergélisol résulte d'un équilibre entre la température au sommet du pergélisol et la température sous le pergélisol, équilibre où n'intervient que la conductibilité thermique vu l'imperméabilité du pergélisol. Dans le pergélisol, la température au sommet de la zone ou l'amplitude annuelle est nulle, est en moyenne de $3,3^{\circ} \mathrm{C}$ plus élevée que la température moyenne annuelle de l'air, (pour 33 sites du Canada, avec cependant un écart type de $1,5^{\circ} \mathrm{C}$, ce qui indique que les écarts sont importants d'après Judge, 1973). Le sol est en effet nettement plus chaud que l'air à $1,5 \mathrm{~m}$ du sol où sont prises les mesures climatiques. De simples données climatiques ne permettent donc pas de calculer la température du pergélisol.

\section{B. La glace du sol}

L'importance fondamentale du pergélisol résulte de ce qu'il permet la conservation de glace en son sein. Lorsque celle-ci se met à fondre, elle entraîne la formation de phénomènes thermokarstiques qui consistent en des affaissements susceptibles d'endommager ou de détruire maisons, routes, et d'altérer considérablement l'environnement.

Pour bien comprendre les phénomènes qui apparaissent, il est indispensable de connaître les différents types de glace du sol qui existent et que nous présentons ci-dessous. 


\section{B.1. LA GLACE DE SÉGRÉGATION}

La glace de ségrégation est formée de lentilles qui apparaissent en cours de gel au sein de sols de granulométrie fine (fig. 4). Elle résulte d'une redistribution de l'eau au sein du sol par suite de sa migration vers le front de gel.

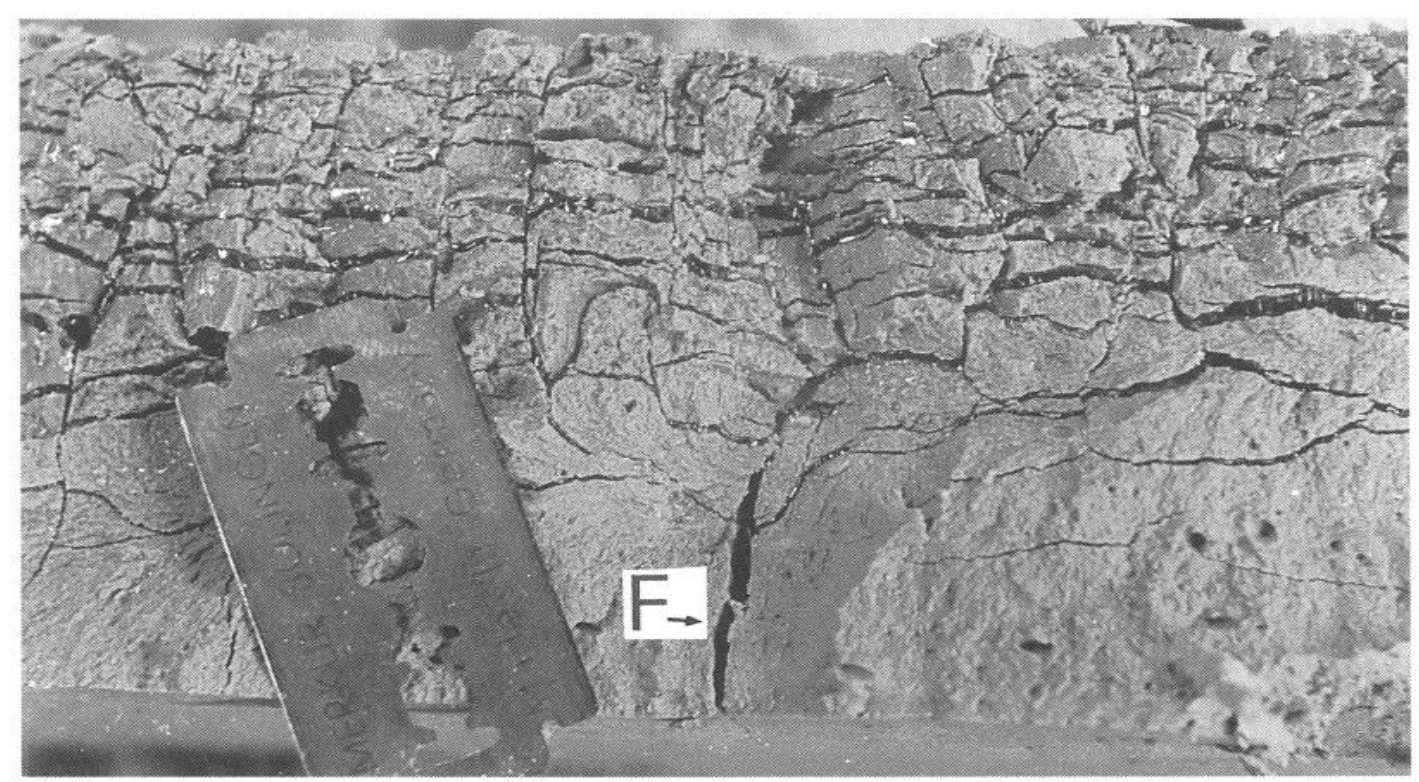

Fig. 4. - Lentilles de glace de ségrégation apparues dans une couche de limon saturée en eau et épaisse de $6 \mathrm{~cm}$ ayant subi en laboratoire un gel lent venu de la surface. Des lentilles de glace de ségrégation principalement parallèles à la surface, c'est-à-dire parallèles au front de gel sont apparues dans la partie supérieure de la masse. La partie inférieure de la masse s'est asséchée et une fente verticale de dessication (F) est apparue (Pissart, 1987).

\section{B.1.1. Mécanismes responsables de sa formation}

En 1929, Taber a montré par des expériences que le soulèvement du sol par le gel n'était pas dû à la simple augmentation de volume de l'eau qui se trouvait dans le sol, mais à l'arrivée d'eau dans la zone de gel. Cette eau vient du sol sous-jacent et se dispose en lentilles de glace de ségrégation.

En 1953, Beskow a expliqué ce phénomène en supposant que la transformation en glace d'une partie de l'eau fortement tenue dans les capillaires du sol par la tension superficielle, a pour effet de diminuer, à l'endroit du gel, le film d'eau adsorbée. Celui-ci a immédiatement tendance à se reconstituer, et l'eau arrive ainsi depuis la zone vers où progresse le gel. Si l'arrivée d'eau compense le refroidissement, l'onde 
de gel reste stationnaire et une lentille de glace de ségrégation grandit en soulevant le sol. Les phénomènes de surfusion jouent un rôle important dans ce mécanisme en empêchant l'eau de se figer dès qu'elle arrive sous $0^{\circ} \mathrm{C}$. En effet, lorsqu'un noyau de congélation apparaît en un endroit et qu'une lentille de glace commence à grandir, la température est à proximité, inférieure à $0^{\circ} \mathrm{C}$. C'est grâce aux phénomènes de surfusion et aussi au fait que l'eau du sol gèle à des températures inférieures à $0^{\circ} \mathrm{C}$ que la circulation de l'eau vers les lentilles de glace peut tout de même s'effectuer. Soulignons que ces mouvements de l'eau engendrent dans le sol sous-jacent des forces de succion considérables.

Il n'y a pas, à ce jour, de théorie unanimement acceptée du mécanisme physique responsable de l'apparition de la glace de ségrégation. Citons deux hypothèses : Chalmers et Jackson (1970) écrivent que les molécules de glace sont à un stade d'énergie plus bas que les molécu-

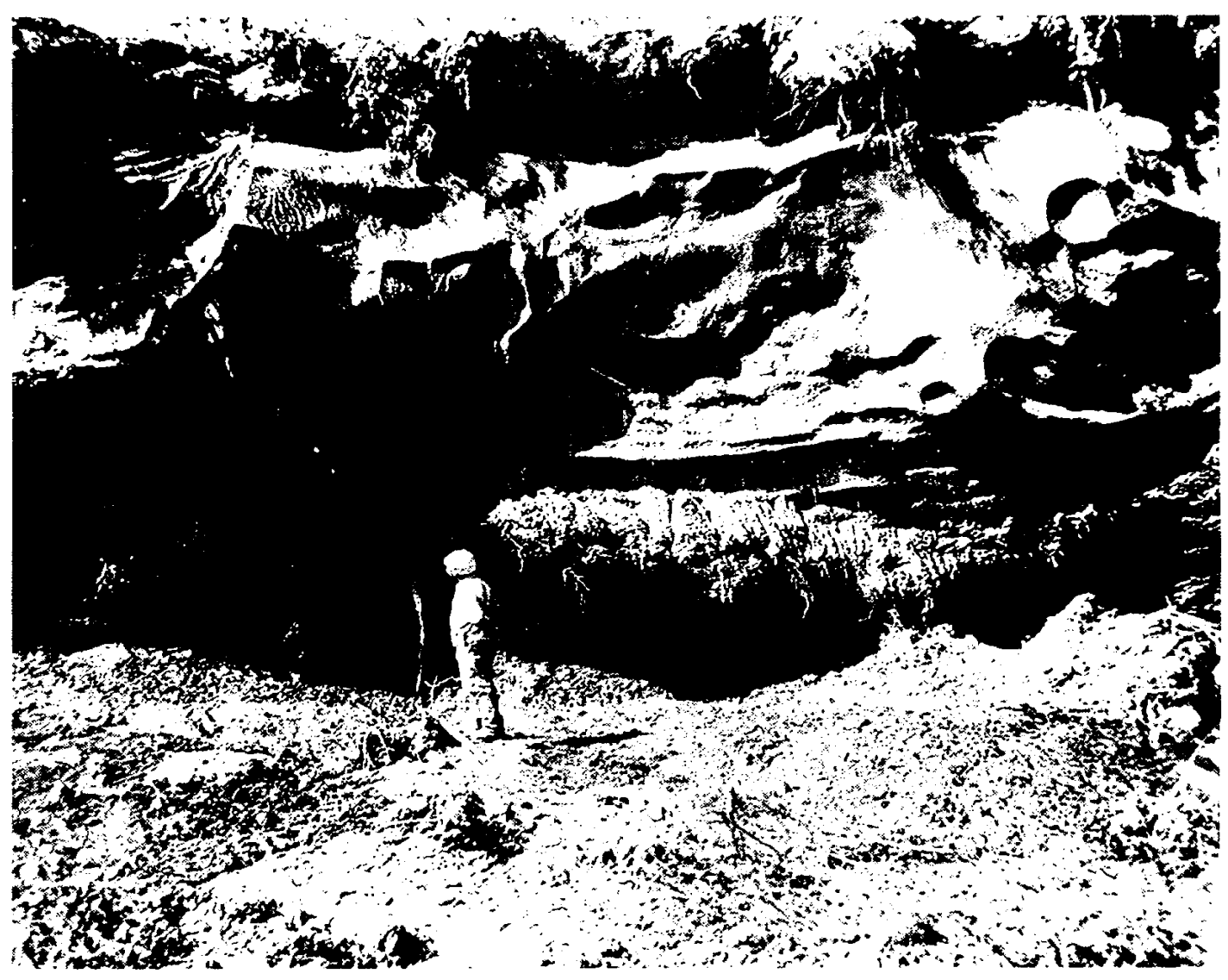

Fig. 5. - La grande quantité de glace de ségrégation et de glace de fente de gel accumulée au voisinage immédiat de la Léna en Yakoutie apparaît clairement par la hauteur de la falaise qui résulte de sa fusion. 
les d'eau de la double couche électrique qui, à son tour, est à un niveau d'énergie plus faible que celui des molécules d'eau libre. Comme le système tend à évoluer vers la plus basse énergie, l'eau migre vers les lentilles de gel (une bonne présentation de cette question est donnée dans Williams, 1982). Par contre, Cass et Miller (1959) soulignent le rôle de la pression osmotique déterminée par la concentration des ions dans la double couche. Comme l'indique Williams (1982), plusieurs forces se combinent pour provoquer la migration de l'eau et nous n'en possédons pas actuellement une intelligence complète.

\section{B.1.2. Conditions requises pour que la glace de ségrégation apparaisse}

Pour que la glace de ségrégation apparaisse, il faut non seulement une certaine humidité dans le sol, mais encore un gel lent. En effet, un refroidissement trop rapide arrête le phénomène en figeant l'eau en train de se déplacer. Par ailleurs, cette glace n'apparaît que si la granulométrie du sol est assez fine pour permettre à l'eau de migrer dans les films capillaires. Précisons en outre que, à moins que l'eau ne soit sous pression dans le sol, la glace de ségrégation ne peut guère se former à plus de $10 \mathrm{~m}$ de profondeur. Plus bas, la pression des terrains surincombants s'oppose au soulèvement du sol par les lentilles de glace et arrête leur croissance.

La glace de ségrégation se dispose principalement en couches parallèles au plan de pénétration de l'onde de gel. Les lentilles ont de moins de $1 \mathrm{~mm}$ à 20 ou $30 \mathrm{~cm}$ d'épaisseur. La glace est évidemment très pure mais peut comprendre quelques inclusions $d$ 'air. L'apparition de lentilles de glace de ségrégation est souvent favorisée par la structure du sol, les hétérogénéités, les ruptures de continuité, les fissures, etc.

Remarquons que les deux conditions d'apparition de la glace de ségrégation (humidité, gel lent) sont bien présentes au sommet du pergélisol. Le gel, au début de l'hiver, commence en effet dans la couche active, non seulement par la surface du sol, mais aussi par le sommet du pergélisol. Des lentilles de glace de ségrégation apparaissent, de ce fait, en ce dernier endroit ; elles peuvent être incluses dans le pergélisol si elles ne fondent pas l'été suivant. Une des causes d'une telle conservation des lentilles de glace formées au sommet du pergélisol peut être l'élévation du sommet du pergélisol par suite de l'accumulation à la surface, de nouveaux sédiments (éoliens, par exemple), d'une 
variation climatique, ou encore d'une modification de la couverture végétale. Cette glace de ségrégation, quand elle est conservée au sommet du pergélisol, est dénommée en anglais "aggrational ice " (Mackay, 1972) et en français "glace sus-pergélisol » (Pissart, 1975) ou «glace d'accroissement» (Harris et al., 1988).

La formation de glace de ségrégation a pour résultat d'accumuler dans le sol des quantités très importantes de glace, quantités qui peuvent être plusieurs fois supérieures en poids à la masse du sol sec au sein duquel elle est apparue (figure 5). D'après Shumsky (1959), la glace de ségrégation est, après la glace ciment dont nous parlerons plus loin, le type de glace dont le volume est le plus important dans la croûte terrestre. Sa concentration au sommet du pergélisol entraîne l'apparition des phénomènes thermokarstiques dès qu'un réchauffement se produit.

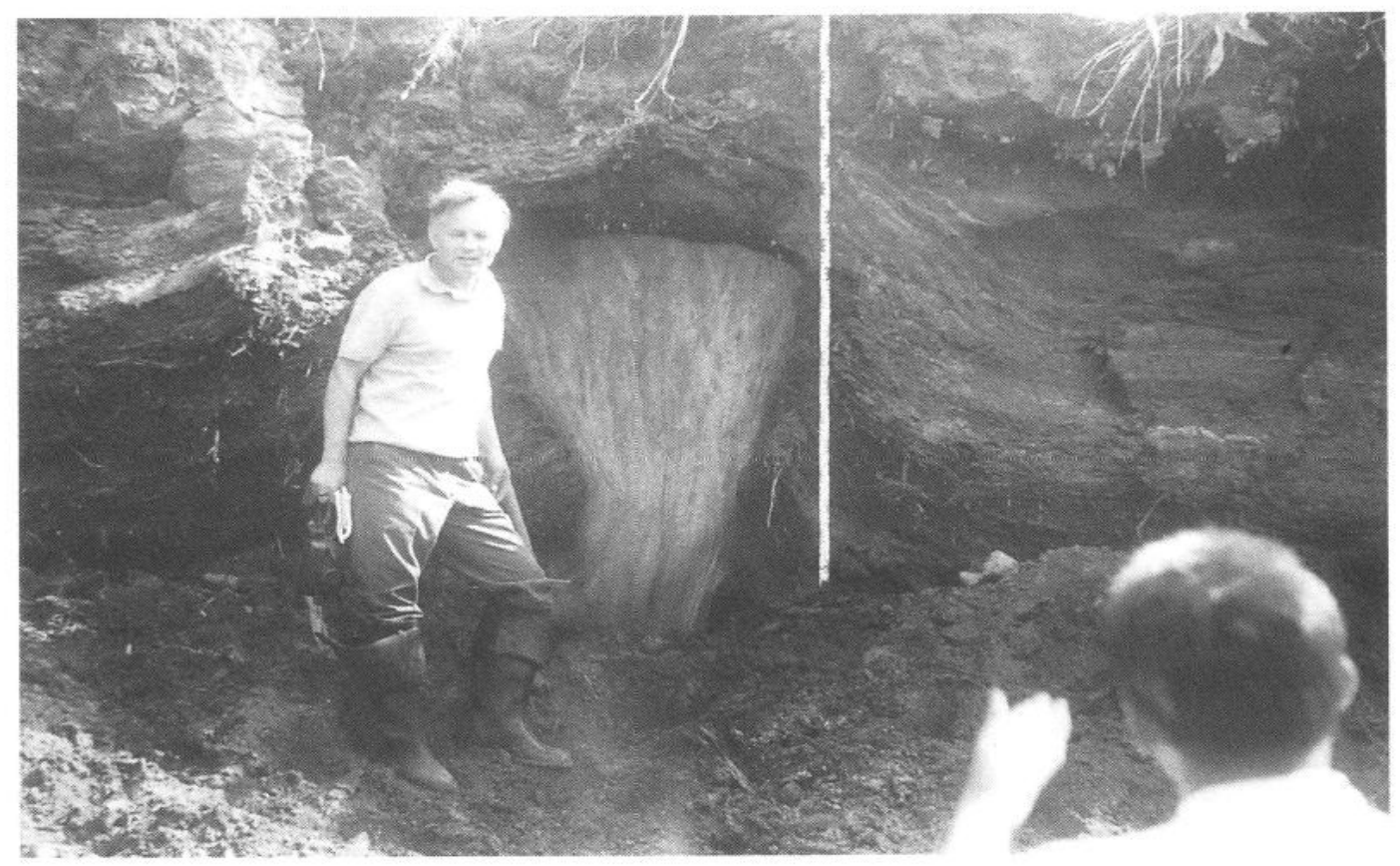

Fig. 6. - Fente de gel à remplissage de glace développée dans les alluvions de la Léna près de Yakutsk (Sibérie). Remarquer la structure en coin de la masse de glace, la structure verticalement litée de la glace et la déformation des couches alluviales de la Léna qui, à l'origine, étaient disposées horizontalement.

\section{B.2. LA GLACE DE FENTE DE GEL}

Les variations de température qui affectent la couche active et le sommet du pergélisol (figure 3) engendrent la formation de fentes de gel. Il s'agit de fissures de rétraction thermique qui se remplissent de glace, 
1. $1^{\text {er }}$ HIVER = Retrait thermique

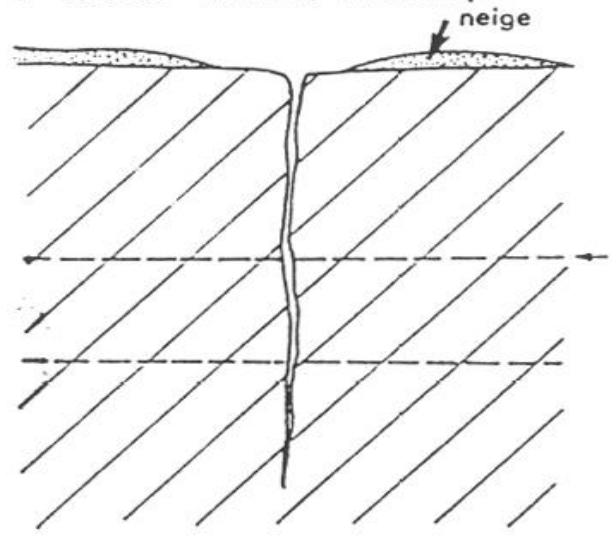

3. 1or ETE = Déformation

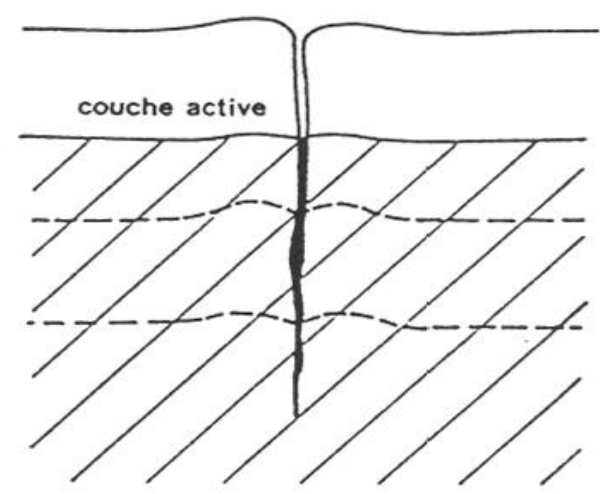

5. $500^{\text {ème }}$ Hiver

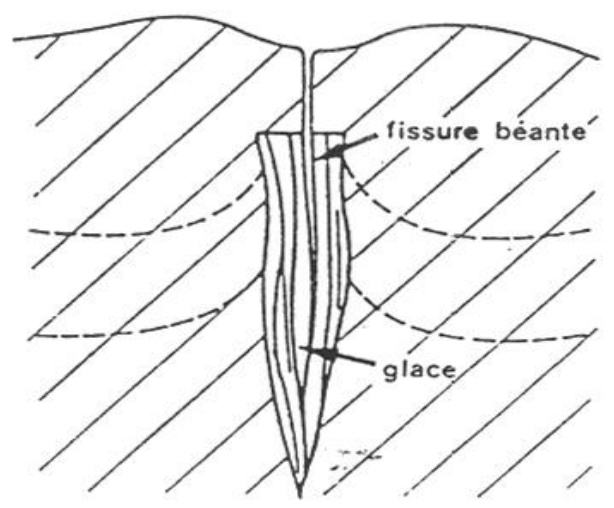

2. $1^{\mathrm{er}}$ PRINTEMPS $=$ Infiltration d eau

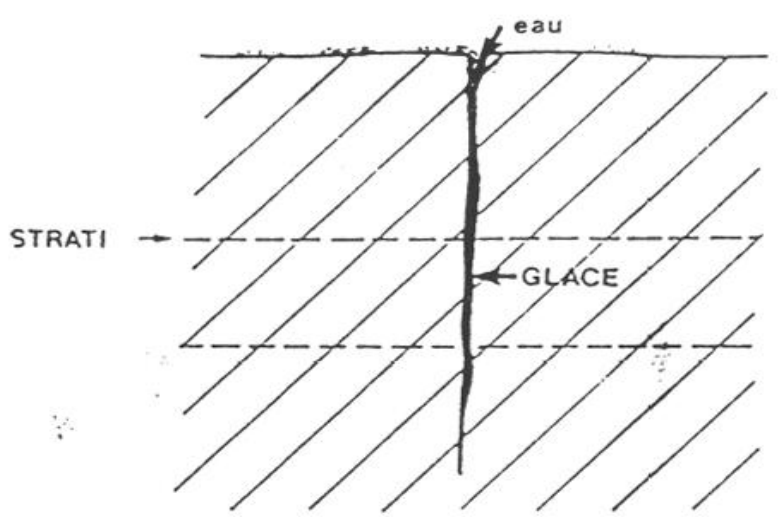

4. $2^{e}$ HIVER $=$ Nouveau retrait thermique

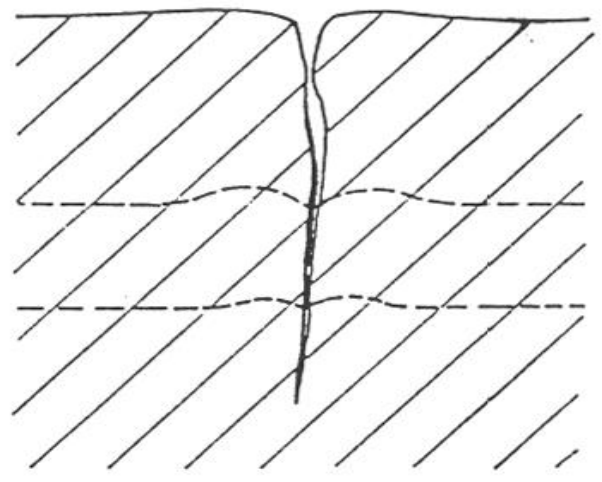

6. $500^{\dot{e} m e}$ Etè

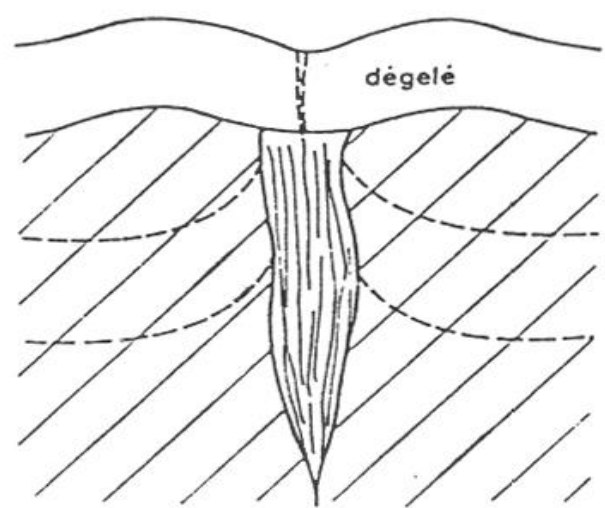

FIG. 7. - Mécanisme d'apparition et de croissance d'une fente de gel à remplissage de glace. Les schémas 1 à 4 montrent l'évolution au début de la formation de la fente de gel; les schémas 5 et 6 montrent son évolution quand la fente de gel est déjà développée. 
de matériel minéral ou d'un mélange des deux. Nous considérerons uniquement ici les fentes de gel remplies de glace (fig. 6) qui poseront problème en cas de réchauffement climatique.

\section{B.2.1. Mécanisme de formation}

Dès 1823, un auteur russe dénommé Figurin expliquait l'apparition de fentes de gel par le retrait thermique du sol pendant l'hiver. Cette théorie est donc ancienne. Elle a été défendue par Bunge (1902) et Leffingwell (1915). Le mécanisme d'apparition de cette glace, illustré sur la figure 7 , est le suivant :

1. Lors des basses températures d'hiver, le sol se refroidissant subit un retrait thermique provoquant sa fracturation en de grands polygones (le plus souvent de quelques dizaines de mètres de diamètre), séparés par des fissures ouvertes larges d'une fraction de $\mathrm{mm}$ à 1 ou $2 \mathrm{~cm}$. Ce phénomène de rupture s'accompagne de bruits (craquements) et aussi de mini-tremblements de terre localement perceptibles.

2. Au moment du réchauffement printanier, la fonte de la neige et de la glace superficielle, comme d'éventuelles précipitations de pluie fournissent de l'eau qui pénètre dans les fissures ouvertes. Descendant dans un sol toujours gelé, cette eau ne tarde pas à se congeler et à remplir de glace la fissure originelle.

3. Le réchauffement estival entraîne le dégel de la couche active surmontant le pergélisol et la fonte de la partie supérieure de la veine de glace. Il provoque, en outre, un réchauffement du pergélisol qui, bien que restant gelé, se dilate et tend à réoccuper les vides apparus lors du retrait thermique. Comme la fissure s'est remplie de glace, le retour des matériaux dans leurs positions originelles est impossible et il se produit une déformation du pergélisol proche de la fissure et, quelquefois, selon les auteurs russes, l'extrusion d'une partie du coin de glace.

4. Lors de la période hivernale suivante, la fissure de glace qui a persisté dans le sol constitue une zone de faiblesse où réapparaît une nouvelle fissure due au retrait thermique. Ainsi d'année en année, par un accroissement annuel de quelques $\mathrm{mm}$, se développe un coin de glace dont la profondeur peut atteindre 12 à $15 \mathrm{~m}$ et la largeur 6 à $8 \mathrm{~m}$. Cette glace présente une stratification verticale caractéristique par suite de l'alternance de couches comprenant des teneurs différentes en inclusions minérales. 
Des fentes de gel apparaissent quelquefois dans des endroits où des dépôts s'accumulent progressivement, comme par exemple des plaines alluviales de remblaiement ou des terrasses où s'accumulent des limons éoliens. Les coins de glace en cours de développement sont alors recouverts chaque année d'une nouvelle couche de sédiments et croissent vers le haut au fur et à mesure où s'élève le sommet du pergélisol. Étant donné que les coins déjà formés constituent des zones de faiblesse rhéologique, c'est toujours aux mêmes endroits que les fissures ouvertes par le gel vont apparaître. Dénommées fentes de gel syngénétiques du dépôt, des structurcs attcignent un développement vertical exceptionnel dépassant quelquefois $50 \mathrm{~m}$ de profondeur. Les structures de ce type sont différentes si la sédimentation s'est produite par épisodes brutaux ou a été progressive. Ces fentes de gel syngénétiques témoignent de ce que le pergélisol a toujours existé au cours du dépôt de ces sédiments.

\section{B.2.2. Les facteurs qui contrôlent la formation des fentes de gel}

Le coefficient de retrait thermique de la glace est cinq fois plus grand que celui de la majorité des silicates, et supérieur à celui du fer (glace : 5,25. $10^{-5}$ ). L'abaissement de la température dans un sol à forte teneur en glace entraîne donc un retrait thermique considérable lors de l'abaissement de la température et explique pourquoi, dans de tels sols, les fentes de gel sont particulièrement bien développées.

L'existence de zones de faiblesse plus ou moins espacées au sein du matériau subissant le retrait joue un rôle fondamental quand le sol commence à se fracturer. Cet élément est évidemment particulièrement difficile à étudier. Mise à part cette question de fragilité initiale, l'espacement des fissures est déterminé par le rapport entre la résistance à la rupture et l'effort de tension qui apparaît dans le sol. Si la résistance à la rupture diminue, les polygones deviennent plus petits. D'autre part, dans des formations contenant peu de glace, les polygones sont généralement plus espacés que dans ceux qui en comprennent beaucoup car comme nous venons de la voir, l'importance du retrait diminue avec le contenu en glace.

L'espacement des fentes de gel paraît contrôlé principalement 1) par la résistance à la rupture du matériau au sein duquel apparaissent les fentes de retrait thermique ; 2) par la profondeur des fissures car celle-ci détermine la largeur de la zone où la tension est relâchée à la suite de l'apparition de la fissure.

Lachenbruch (1966) a recherché une expression mathématique qui représenterait le phénomène de rupture du sol par le gel. Il conclut 
qu'une telle expression ne peut être trouvée car le pergélisol ne se comporte, ni comme un solide élastique, ni comme un corps visqueux. Dans le premier cas, en considérant la vitesse de propagation des ondes soniques et en appliquant les paramètres qui la définissent, les ruptures se produiraient pour des variations de température de seulement 2 à $3{ }^{\circ} \mathrm{C}$. Les faits d'observation montrent que cela ne correspond pas à la réalité. S'il s'agit d'un solide visqueux, la vitesse de chute de la température intervient avec la valeur absolue de la température. Effectivement, les ruptures paraissent se produire quand l'abaissement de la température est brutal. Lachenbruch (1966) souligne que les réponses à ces deux facteurs, à savoir la valeur absolue de la température et la vitesse d'abaissement de la température, ne sont pas linéaires mais complexes.

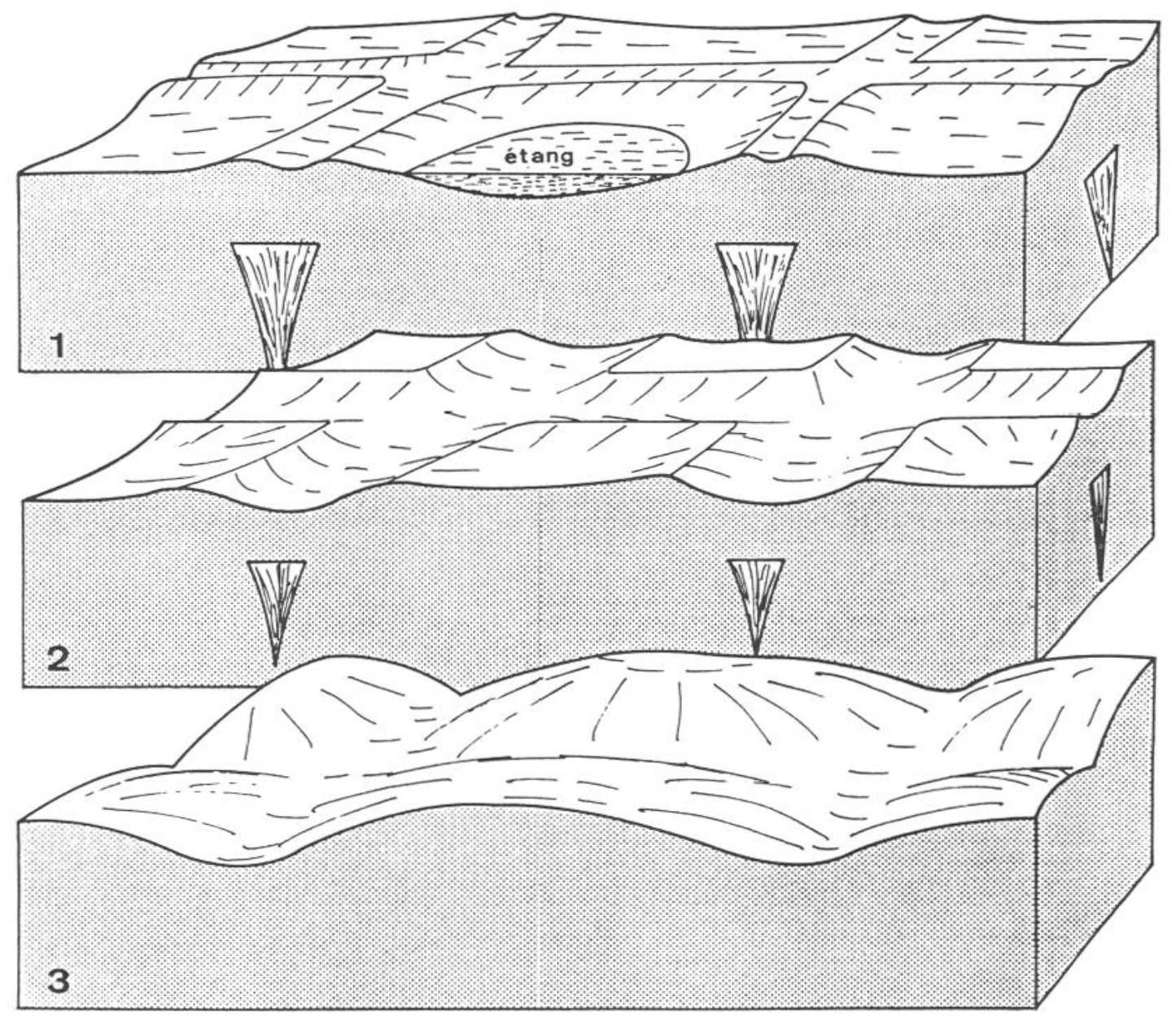

Fig. 8. - Schéma montrant l'aspect en surface d'un réseau de fentes de gel à centres déprimés (1), évoluant par fusion des coins de glace vers un réscau de polygones à centres bombés ( 2 et 3 ). 
Il convient de préciser enfin, pour bien comprendre le phénomène que, vu les différences de température qui existent dans le sol en été et en hiver, les propriétés mécaniques ne sont pas les mêmes au moment où, au refroidissement, se développent les tensions et, où au réchauffement, se produisent les compressions.

B.2.3. Apparence à la surface du sol de la glace de fente de gel: les polygones de fente de gel

Par la déformation caractćristique des couches sédimentaires où elles apparaissent, les fentes de gel en croissance apparaissent clairement à la surface du sol. À proximité de chaque fente de gel, le sol est soulevé et, en terrain horizontal, apparaît un réseau de polygones à centres déprimés (fig. 8.I). Si à la suite d'un réchauffement climatique ou d'une modification de la surface du sol, les coins de glace commencent à fondre apparaissent alors des polygones à centres bombés (fig. $8.2 \mathrm{et}$ 8.3).

\section{B.3. La GLACE D'INJECTION ET LES PINGos}

Un troisième type de glace du sol, beaucoup moins répandue que les deux précédentes, est la glace d'injectior. Elle donne naissance à des buttes spectaculairess qui peuvent dépasser $50 \mathrm{~m}$ d'élévation et qui sont connues dans la littérature occidentale. sous le nom esquimau de "pingo »

\section{B.3.1. Mécanisme de formation des pingos}

Depuis la publication de Porsild en 1938, deux systèmes génétiques différents sont proposés pour expliquer l'origine des pingos. Ils sont dénommés "système fermé » et «système ouvert ». Nous les examinerons ci-dessous.

a. Les pingos nés en système fermé

Les pingos du delta du Mackenzie sont actuellement les mieux connues, principalement grâce aux recherches de Mackay (1979). Aussi ce type de pingo est-il dénommé "pingo du Mackenzie " (fig. 9).

Ces formes, comme celles de la Yakoutie centrale qui ont la même origine, sont presque toujours localisées à l'emplacement d'anciens lacs. C'est le cas de $98 \%$ des 1380 pingos comptés par Stager (1956) dans le delta de Mackenzic. Ces lacs jouent en effet un rôle détermi- 


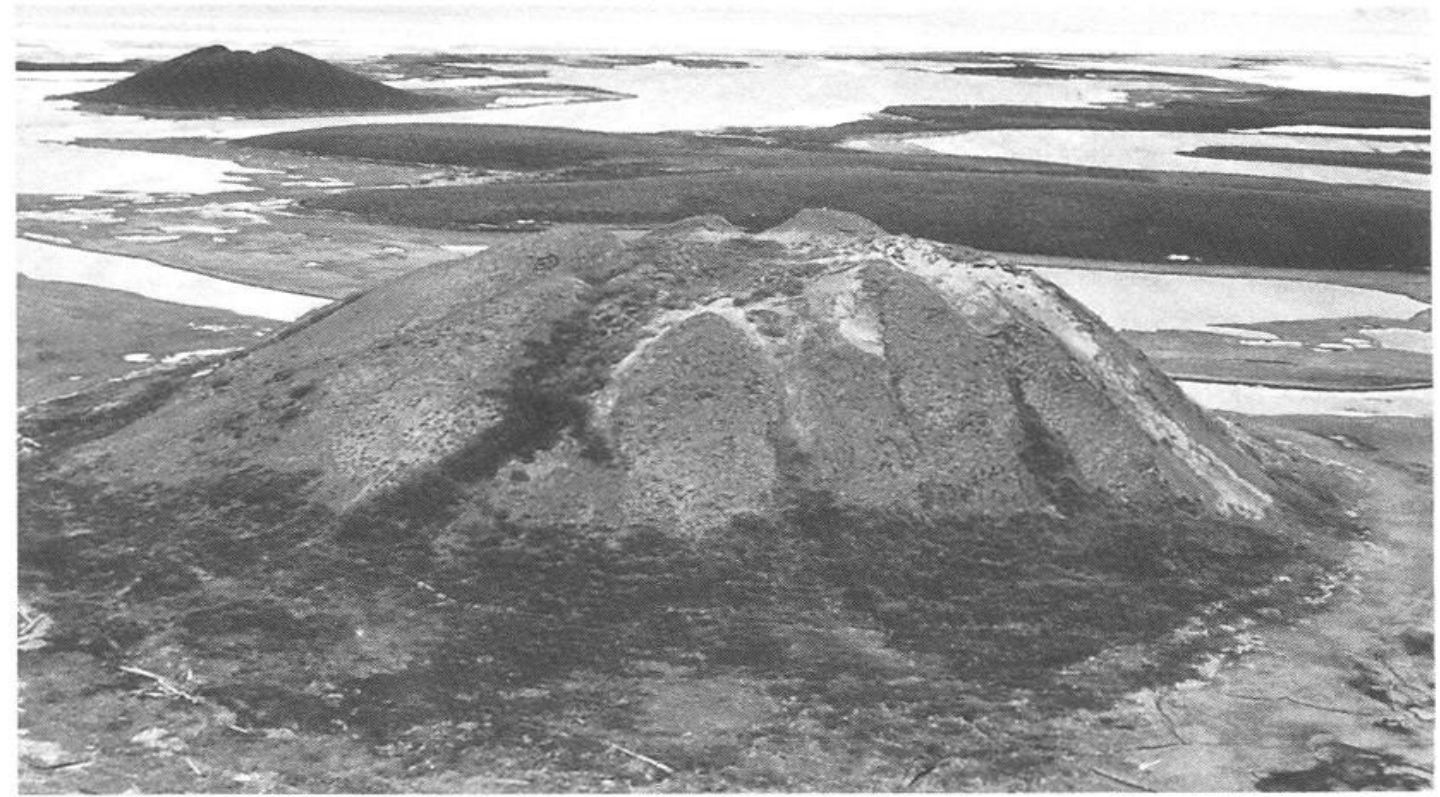

Fig. 9. - Photo de l'Ibyuk pingo, près de Tuktoyaktuk dans le delta du Mackenzic. Avec $49 \mathrm{~m}$ d'élévation, il s'agit du pingo le plus haut de la région. Il s'élève encore de nos jours selon Mackay (1976) de 2,3 cm par an. Son âge'serait d'environ 1000 ans. Au fond à gauche est visible un autre pingo.

nant dans le mécanisme qui leur donne naissance, comme nous l'expliquons ci-dessous.

Dans des régions où le pergélisol atteint plusieurs centaines de mètres d'épaisseur, un talik (zone non gelée) existe sous les lacs qui sont assez profonds. Cette zone non gelée s'explique d'une part parce qu'en hiver, à l'emplacement de ces lacs, le gel n'atteint pas le fond; d'autre part, parce qu'en été l'eau des lacs, échauffée par les rayons solaires qui y pénètrent, a une température plus élevée que celle du sol voisin. Aussi la température moyenne du fond du lac est nettement plus élevée que la température de la surface du sol autour de celui-ci.

$\mathrm{Si}$, à la suite de la baisse du niveau du lac ou d'un apport de sédiments, l'épaisseur de la nappe d'eau diminue, le fond du lac commence à geler. Ainsi, apparaît sous le fond de l'ancien lac une poche de sédiments non gelés, entourée de toutes parts de sédiments déjà pris par le gel (fig. 10). Porsild (1938) comparait le phénomène avec ce qui se produit dans une bouteille remplie de lait et soumise au gel : l'augmentation de volume qui accompagne le gel de l'eau, détermine dans le système fermé que constitue la poche de sédiments non gelés, une augmentation de pression qui va provoquer une injection d'cau vars la surface.

Quelquefois, cette eau injectée peut arriver à la surface où elle s'écoule ou si le phénomène se passe en hiver, elle s'accumule en une 

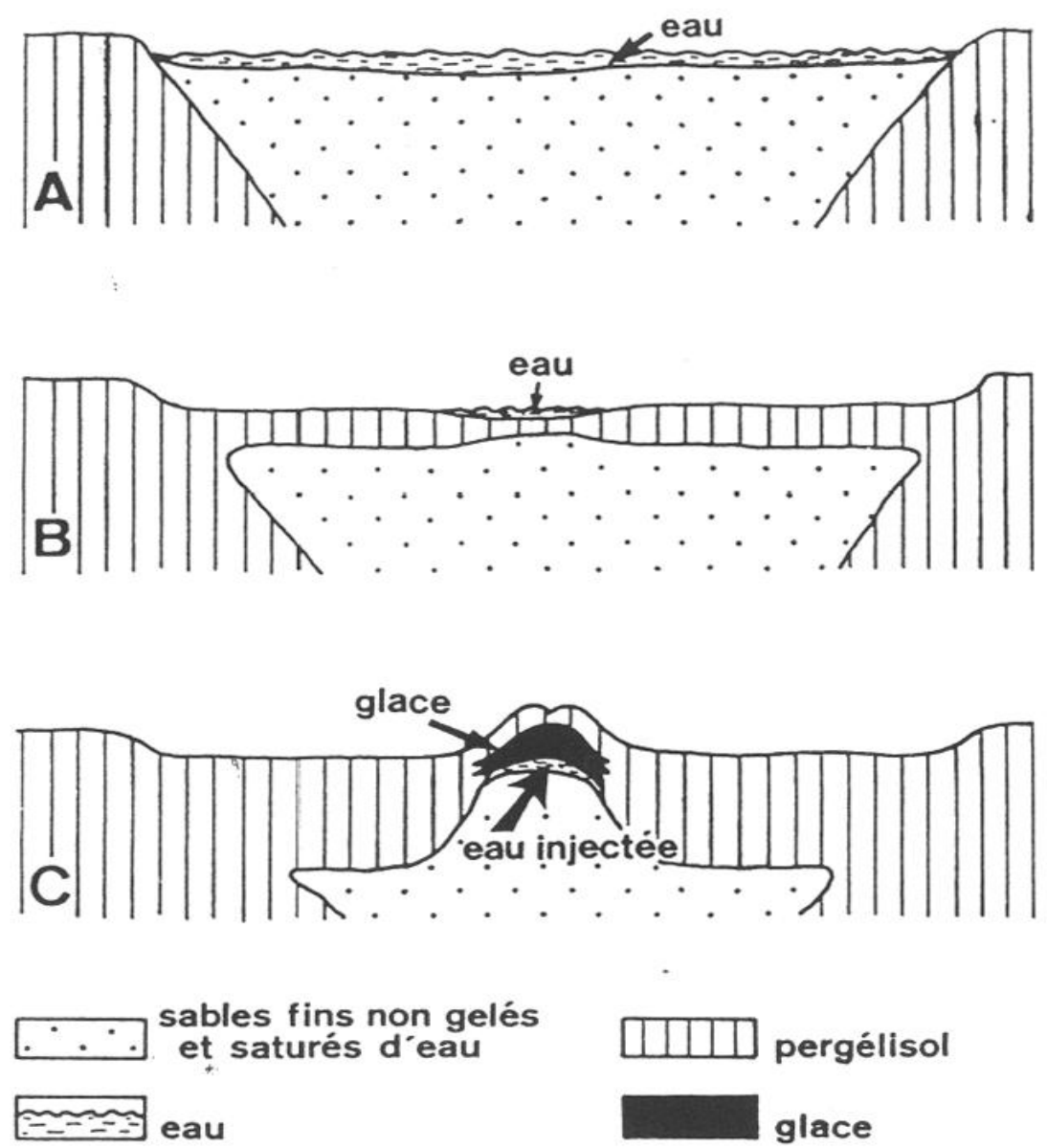

FIG. 10. - Schéma montrant l'origine des pingos du type Mackenzie, c'est-àdire apparaissant en système fermé. En A, sous un lac existe un talik (poche non gelée) entourée d'un pergélisol. En B, le niveau du lac s'est abaissé et le fond du lac commence à geler mettant l'cau du sol sous pression; en C, de l'eau est injectéc sous un pingo, cette eau en gelant va devenir de la glace d'injection et être intégrée au noyau de glace du pingo (d'après Mackay, 1979).

masse de glace (dénommée icing) (Shumsky, 1959 ; Mackay, 1977). Le plus souvent cependant, l'eau est retenue dans le sol à faible profondeur, sous une couche de sédiments de quelques mètres qui est soulevée. Cette poche d'eau y est, par la suite, congelée en donnant ce que l'on appelle de la glace d'injection, c'est-à-dire de la glace résultant de la congélation d'une masse d'eau injectée dans le sol.

Des pingos en système fermé naissent parfois à la suite de la reprise par le gel de taliks (parties du sol non gelées) apparus soit sous des rivières, soit à la suite d'un mouvement du niveau de la mer, mais ils sont par le mécanisme qui leur donne naissance, identiques aux pingos du delta de Mackenzie que nous venons de décrire (Pissart, 1967 ; Pissart et French, 1976). 


\section{b. Les pingos nés en système ouvert}

Pour les pingos nés en système ouvert, comme dans le cas de pingos nés en système fermé, l'eau mise sous pression, est injectée dans le sol surincombant. Toutefois, il ne s'agit plus ici de pression cryostatique (c'est-à-dire due au gel) mais d'une simple pression hydrostatique due à la mise en charge d'une nappe aquifère en relation avec une différence d'altitude.

Dans le cas le plus fréquent, c'est-à-dire qui est décrit aussi bien en Sibérie (Shumsky, 1959) qu'au centre de l'Alaska (Holmes, Hopkins and Foster, 1968) et au Canada dans le Yukon (Hughes, 1969), ces formes apparaissent dans la zone de pergélisol discontinu. L'eau circule alors, comme le montre la fig. 11, sous le pergélisol qui est relativement peu épais. L'alimentation de la nappe est réalisée depuis la surface par les espaces libres de pergélisol.

S
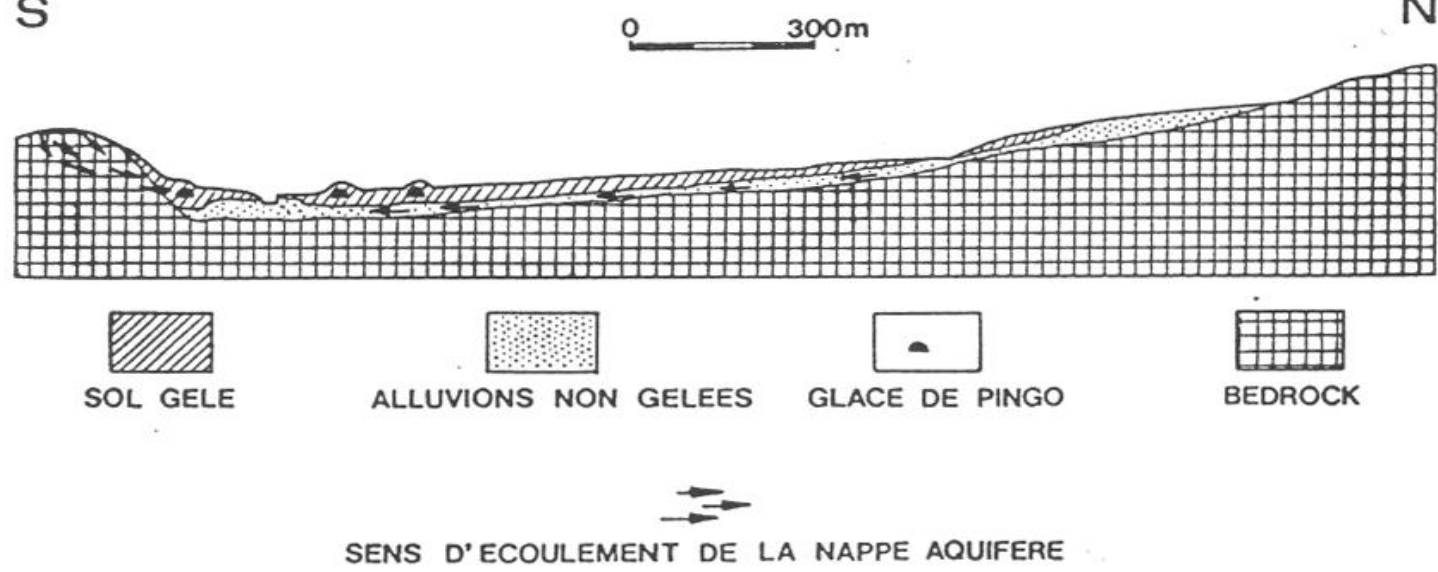

FIG. 11. - Schéma illustrant l'origine des pingos nés en système ouvert (d'après Holmes. Hopkins et Foster, 1969).

Ces pingos apparaissent habituellement au pied des versants près du contact avec les fonds de vallée. Ils se forment habituellement sur des pentes modérées à moyennes (2 à $26^{\circ}$ au Yukon, selon Hughes, 1969) en soulevant des dépôts de pentes remaniant débris du bedrock, dépôts éoliens et colluviaux. Ces pingos sont toujours très dispersés.

Au Groenland et au Spitzberg, des pingos de ce type ont été décrits dans des zones de pergélisol continu. Ainsi, l'ensemble des données réunies par Liestol (1977) pour le Spitzberg montre que l'épaisseur du pergélisol varie de 75 à $450 \mathrm{~m}$ dans la région de Svalbard, où il a décrit près de 70 pingos en système ouvert. 


\section{B.3.2. Importance de la glace d'injection}

Les pingos constituent des formes spectaculaires mais très localisées résultant de l'accumulation de glace dans le sol. Il n'était pas possible de faire l'inventaire des types de glace du sol de l'Arctique sans en faire mention. Étant donné leur relative rareté, leur disparition à la suite d'un réchauffement climatique n'aurait que des conséquences très localisées.

\section{B.4. LeS AUTRES TYPES DE GLACE DU SOL}

À côté des trois types de glace du sol que nous venons de décrire existe aussi la "glace ciment " de Shumsky (1959), équivalente de la «pore ice " de Mackay (1972), laquelle résulte de la congélation de l'eau à l'endroit où elle se trouve dans le sol au moment du gel, et par exemple dans les espaces entre les cailloux et les grains de sable. Shumsky (1959) distingue, en outre, la glace apparue dans de larges cavités originelles de la roche où l'eau se trouvait libre avant le gel, et il l'appelle "glace filonienne constitutionnelle ». Cette distinction à l'avantage de souligner l'aspect très différent que présente cette glace suivant qu'elle est apparue entre les grains d'un sable par exemple ou dans une cavité du bedrock. De toutes les glaces du sol que nous avons considérées, la glace ciment est celle dont le volume est le plus grand dans la croûte terrestre.

Lcs consćquences susceptibles de résulter de la fusion de cette glace ciment ne sont pas importantes. En effet, en se congelant l'eau a changé d'état mais ne s'est pas déplacée, ni surtout accumulée en certains endroits. La fusion de cette glace n'entraînera donc aucun tassement, ni aucune sursaturation du sol. Il n'y aura aucune conséquence si le pergélisol au sein de laquelle elle est conservée disparaît.

Il reste à mentionner l'existence au sein du pergélisol de glaces enfouies : restes de glaciers recouverts de moraines, glaces de rivières, de lacs ou accumulations de neige recouvertes de sédiments. Ces masses de glace peuvent être très importantes sans que leur existence ne soit nécessairement perceptible à la surface. Heureusement relativement rare, cette glace très massive entraîne lors de sa fusion une perturbation considérable de la surface du sol. 


\section{B.5. IMPORTANCE ET RÉPARTITION DE LA GLACE DU SOL}

Comme nous nous sommes efforcés de le faire apparaître dans la présentation que nous avons donnée ci-dessus, la glace de ségrégation et la glace de fente de gel sont extrêmement fréquentes à la partie supérieure du pergélisol. Ces glaces sont particulièrement abondantes dans les sols fins des dépressions humides. Elles sont moins fréquentes ou absentes dans les sédiments grossiers, sur les sommets et évidemment dans les roches consolidées. Les fentes de gel actives qui apparaissent clairement en surface par l'apparition des polygones de toundra, les pingos et les palses qui souvent peuvent être aisément reconnus, sont autant de preuves de la présence de glace et de l'existence d'un pergélisol. Ce n'est toutefois pas avec ces observations que l'on peut cartographier l'extension et l'importance de la glace du sol. Son existence est souvent seulement perceptible quand elle fond et que des phénomènes thermokarstiques apparaissent.

En conséquence, en l'absence de sondages, il est souvent impossible de dire si de la glace existe ou non dans le pergélisol. Il est tout aussi impossible dans le domaine du pergélisol discontinu, de reconnaître par des phénomènes de surface si localement un pergélisol existe, ce qui signifie qu'il existe un risque sérieux de présence de glace du sol.

Il convient encore d'insister sur le fait que les glaces de ségrégation et de fentes de gel sont particulièrement abondantes au sommet du pergélisol. Tout épaississement de la couche active qui se produirait à la suite d'un réchauffement climatique va entraîner la fusion de la partie sommitale de cette glace.

\section{Complexité des relations existant entre la température de l'air et la température au sommet du pergélisol}

C'est évidemment le climat qui contrôle essentiellement l'extension du pergélisol. Les premiers îlots de pergélisol sont observés lorsque la température moyenne annuelle est de l'ordre de $-1,1^{\circ} \mathrm{C}$. La courbe annuelle de $0^{\circ} \mathrm{C}$ ne correspond donc pas à l'apparition du pergélisol et cela parce que la température de la surface du sol est supérieure à celle de l'air. Les courbes isothermes que donnent les cartcs climatologiques, sont obtenues en effet par des mesures réalisées dans des abris météorologiques à $1,50 \mathrm{~m}$ au-dessus du sol. 
La température dont la valeur correspond effectivement à l'extension du pergélisol est la température moyenne annuelle au sommet du pergélisol, endroit où évidemment les mesures sont très rares. Entre la température de l'air et la température au sommet du pergélisol, des différences importantes existent. S'interposent en effet entre les deux, non seulement toute la couche active mais encore la couverture végétale et la couverture temporaire de neige. En outre, au sein de la couche active interviennent aussi de manière déterminante les changements de phase de l'eau qui se produisent au cours de l'année, à savoir les actions de gel et de dégel de l'eau, tout comme les phénomènes d'évaporation.

L'importance des différents facteurs que nous venons d'énumérer est telle que le passage du pergélisol discontinu au pergélisol continu correspond seulement à la couche isotherme annuelle de $-6,6^{\circ} \mathrm{C}$. Cette valeur signifie donc que des sédiments non gelés existent en des endroits où la température moyenne annuelle de l'air est inférieure à $-6^{\circ} \mathrm{C}$.

Parmi tous les éléments que nous venons d'évoquer, le facteur le plus important qui contrôle la température du sol est sans aucun doute la couverture de neige qui recouvre le sol pendant l'hiver. Cette couverture neigeuse est un excellent isolant thermique. Une diminution de son épaisseur pendant l'hiver surtout si son épaisseur est inférieure à $80 \mathrm{~cm}$ va entraîner un refroidissement important du sol ; inversement, une épaisseur plus grande de neige détermine un réchauffement. Dans le même ordre d'idées, une répartition différente des précipitations neigeuses au cours de l'année peut, pour un volume de précipitations identique, provoquer un réchauffement ou un refroidissement du sol selon que les précipitations tombent soit au début de l'hiver, soit plus tard dans cette saison. La distribution inégale de la couverture neigeuse qui est déplacée par le vent (car la neige reste poudreuse longtemps dans ces régions froides), va de la même manière contrôler la répartition du pergélisol. Celui-ci peut être absent où la neige s'accumule, alors qu'il est bien développé à quelques dizaines de mètres où la neige est emportée par la déflation.

La végétation joue aussi un rôle très complexe. Elle protège généralement plus le sol de la chaleur que du froid. Sous la forêt, l'ombre des arbres protège le sol du réchauffement pendant l'été ; l'hiver, les arbres peuvent retenir une partie des précipitations neigeuses et permettre un refroidissement du sol plus intense. Mais en diminuant la circulation de l'air, en augmentant l'évaporation, en modifiant l'action du vent, l'influence de la couverture végétale est très complexe et son action sur la température du sol n'est généralement pas prévisible. 
Différentes associations végétales, le désert polaire, la toundra et la taïga, se partagent la majorité des régions où existe un pergélisol. Ce qui détermine les limites d'extension des associations végétales dans les régions froides est la température du mois le plus chaud. Ainsi, la forêt disparaît au nord lorsque la température moyenne du mois le plus chaud s'abaisse sous $10^{\circ} \mathrm{C}$. La répartition des associations végétales ne fournissent donc pas d'observations permettant de cartographier le pergélisol.

Comme nous l'avons expliqué précédemment en parlant des pingos en système fermé, la présence de nappe d'eau, d'un lac et d'une rivière entraîne un réchauffement du sol sous-jacent et engendre la disparition du pergélisol si la nappe d'eau ne gèle pas jusqu'au fond.

Le relief (en ce qu'il détermine des différences d'exposition aux rayons du soleil), le drainage du sol (en relation avec le taux d'humidité), la nature du sol (par exemple la mauvaise conductibilité thermique de la tourbe sèche l'été et sa bonne conductibilité lorsqu'elle est humide l'hiver), le gradient géothermique et l'histoire géologique (en ce qui concerne spécialement l'extension des glaciers, des mers et des lacs), tous ces facteurs interviennent également pour déterminer les caractères et l'extension du pergélisol. L'ensemble de ces éléments explique que la température moyenne annuelle du sol varie de plusieurs degrés centigrades sur une distance de quelques centaines de mètres.

\section{Les phénomènes thermokarstiques}

L'ensemble des facteurs que nous venons d'énumérer montre toutes les influences locales que subit le pergélisol et souligne combien il est difficile de cartographier en détail sa distribution lorsqu'il est discontinu. Ces facteurs indiquent aussi qu'un pergélisol peut localement apparaître ou disparaître (indépendamment de tout changement climatique), si un des facteurs que nous avons mentionnés précédemment, est altéré. Pour illustrer par un exemple les modifications qui peuvent se produire, la figure 12 montre les conséquences d'une disparition locale de la forêt, ce qui peut arriver naturellement à la suite d'un incendie ou le plus souvent à la suite d'un défrichement anthropique. La disparition de la forêt entraîne le développement de la couche active aux dépens du sommet du pergélisol ct donc la fusion, à la fin de l'été de la partie supérieure des coins de glace de fente de gel. Cette fusion provoque localement l'affaissement de la surface du sol, tandis que l'eau prove- 


\section{FRAGILITE DU PERGELISOL}

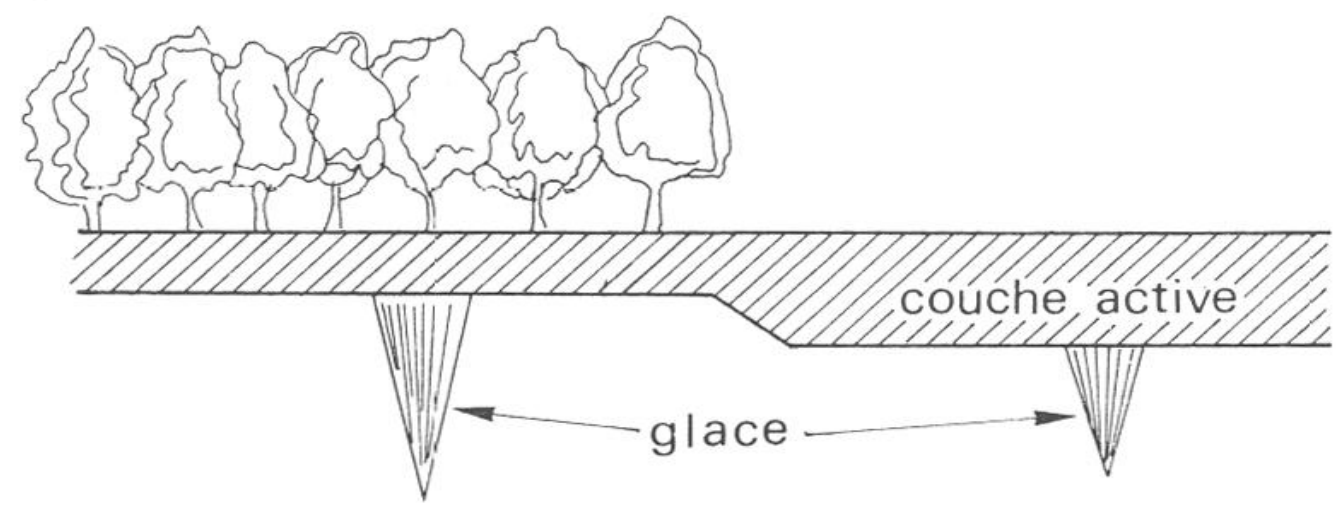

INCENDIE ET DESTRUCTION DE LA FORET

1 an après I'incendie

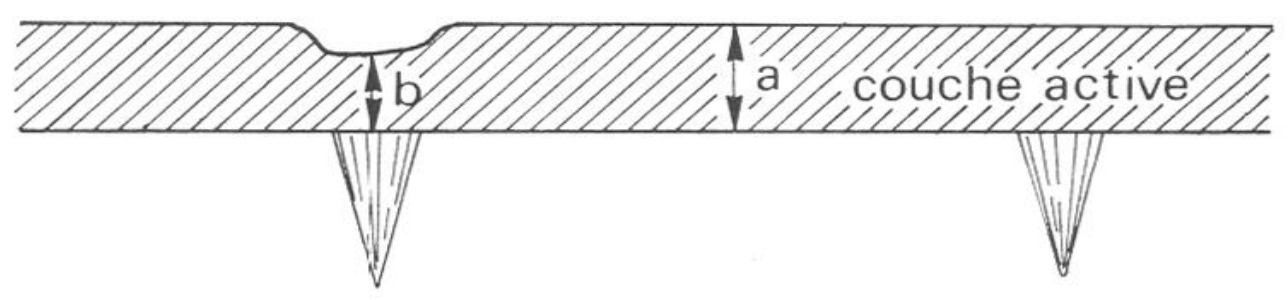

3 ans après I'incendie

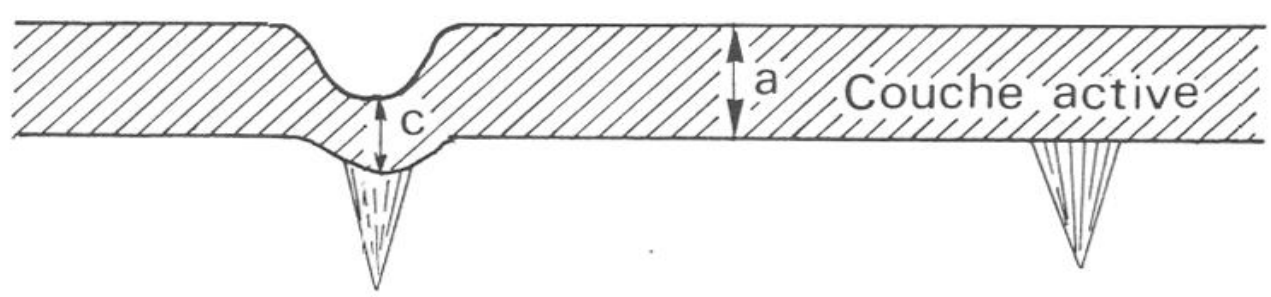

FIG. 12. - Conséquence de la disparition de la forêt au-dessus d'un réseau de fentes de gel à remplissage de glace : la couche active s'épaissit et des dépressions thermokarstiques apparaissent. Celles-ci peuvent, en quelques années, faire fondre toute la glace de fente de gel en formant des dépressions de plusieurs mètres de profondeur. 


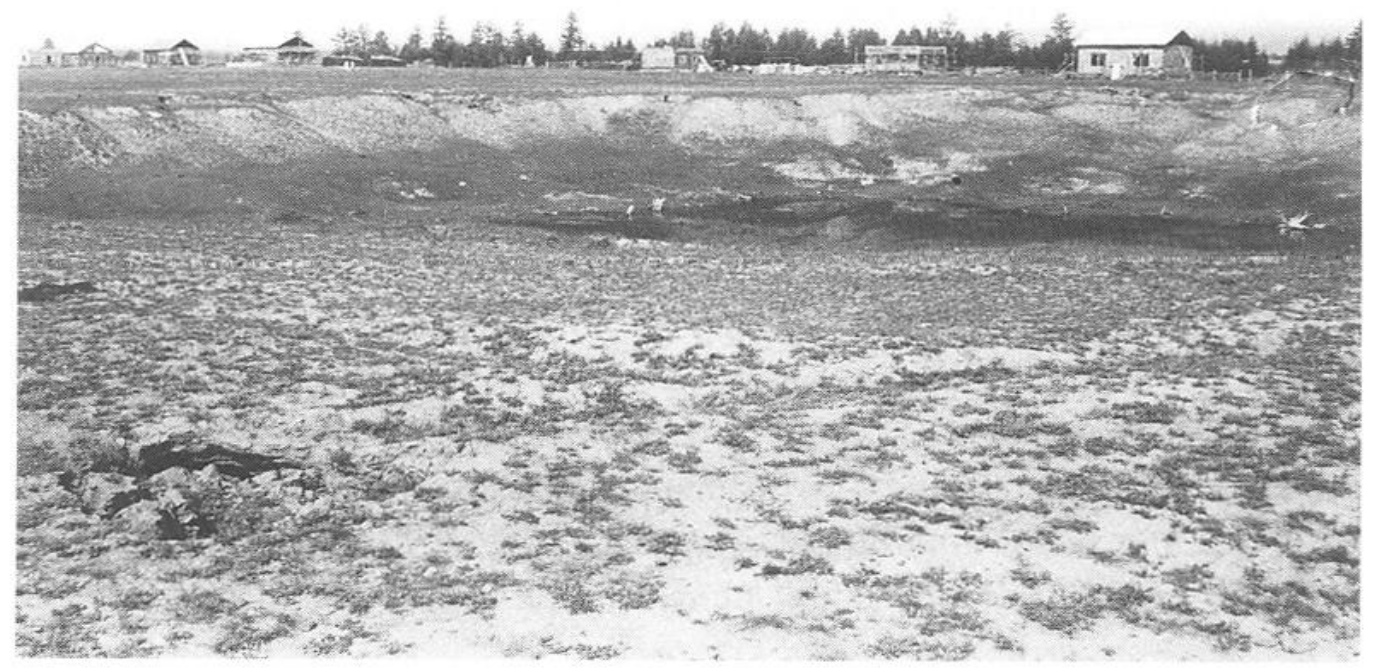

Fig. 13. - Vue partielle d'une grande dépression thermokarstique près de Yakutsk en Sibérie. A l'avant plan, apparaît un petit effondrement récent qui est le début d'une nouvelle cuvette de thermokarst.

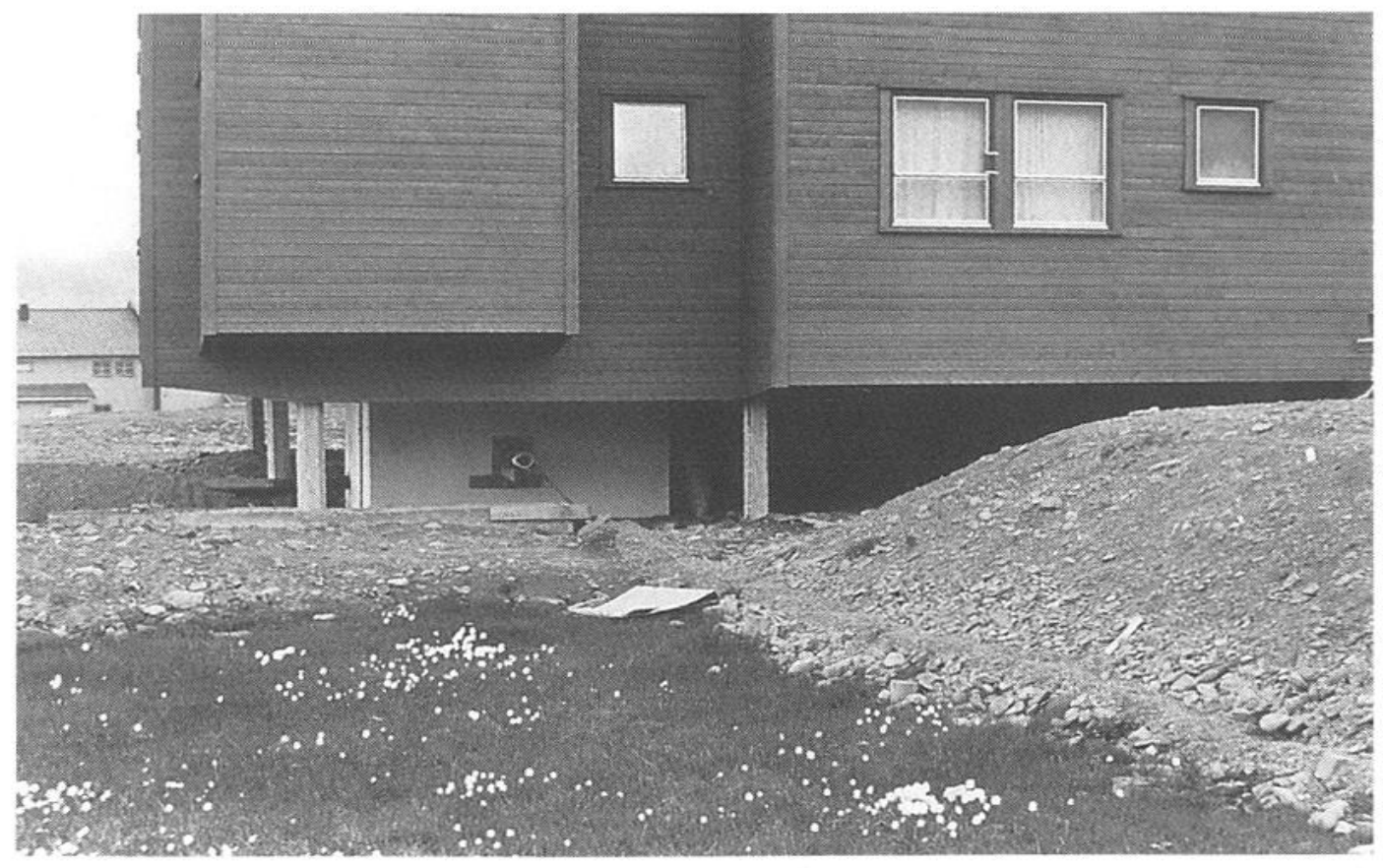

Fig. 14. - Les méthodes de protection contre la fusion du sol sont illustrées par cette photo prise à Longyearbyen au Spitzberg : constructions sur pilotis et remblaiement du sol avec des graviers et des sables. 
nant de la fusion du coin de glace est évacuée. L'épaisseur de la couche active qui surmonte le coin de glace n'est en conséquence pas plus grande après cette fusion, et l'été suivant, la fonte du coin de glace va se poursuivre. Ainsi le phénomène continuera d'année en année, jusqu'à ce que, par descente des matériaux voisins de la fente de gel ou par reconstitution de la couverture végétale, le dégel n'atteigne plus le sommet du coin de glace. Un tel phénomène illustre bien qu'une faible modification de la surface du sol peut entraîner en plusieurs années des affaissements thermokarstiques tout à fait considérables et destructeurs pour les constructions, routes, chemin de fer, pistes d'aviation,... que l'homme a établi dans ces régions froides (fig. 13).

L'homme parvient à se protéger contre le développement de ces phénomènes thermokarstiques en prenant des mesures pour que le dégel n'atteigne pas le sommet du pergélisol. Ces mesures consistent : 1) à ne pas décaper la surface du sol et ne rien en enlever avant de construire ; 2) à y accumuler des matériaux grossiers (sables et graviers) au sein desquels de la glace de ségrégation ne peut apparaître ; 3) à organiser (en posant des tuyaux au sein de ces sédiments) une circulation d'air froid qui est entretenue au cours de l'hiver, tandis qu'elle est arrêtée pendant l'été lorsque la température de l'air est élevée ; 4) à construire sur des pilotis profondément ancrés au sein du pergélisol ; 5) à installer des siphons thermiques utilisant des changements de phase vapeur/liquide qui fonctionnent en hiver uniquement en entraînant un refroidissement du pergélisol (fig. 14).

En dehors d'un réchauffement climatique, les mesures que nous venons d'énumérer suffisent à empêcher tout réchauffement du sommet du pergélisol et tout effondrement du sol à la suite des activités humaines. Il semble que ces procédures seront suffisantes pour empêcher ou limiter les effondrements sous les constructions de la majeure partie de la zone du pergélisol pour un réchauffement climatique élevant la température moyenne annuelle de 2 à $4^{\circ} \mathrm{C}$ et se produisant en hiver (Vyalov et al, 1993). Le problème subsiste cependant si le réchauffement est plus important, ou encore pour les parties méridionales du pergélisol discontinu où le réchauffement du sol se produira de toute manière, que le réchauffement climatique ait lieu en été ou en hiver. Enfin pour des équipements comme les routes où les interventions sur tout le tracé seront impossibles, la fusion de la glace du sol pourra être très destructrice.

Les phénomènes thermokarstiques constituent les phénomènes spectaculaires les plus évidents qui résultent de la fusion de la glace contenue dans le pergélisol, mais d'autres phénomènes peuvent être attendus comme de nombreux glissements de terrains, des modifications du drainage, etc. 


\section{E. Le réchauffement climatique des régions arctiques : les incertitudes et les difficultés d'en prévoir les conséquences}

Les nombreux modèles climatiques du réchauffement prévu à la suite de l'augmentation des gaz à effet de serre, prédisent l'augmentation la plus importante de la température dans les régions polaires (Hansen et al., 1988 ; Berger, 1992 ; Nelson et al, 1993). Les chiffres avancés sont tous différents, mais le modèle de Hansen et al. prévoit que pour un accroissement possible de $1^{\circ} \mathrm{C}$ en 2010 pour toute la terre, la température moyenne dans les régions à pergélisol s'élèverait entre 1 et $3{ }^{\circ} \mathrm{C}$.

D'après les modèles, le réchauffement annoncé se produira essentiellement l'hiver et très peu l'été. Un réchauffement des étés entraînerait dans toute la zone où existe un pergélisol, un accroissement d'épaisseur de la couche active et donc, avec la fusion de la glace accumulée au sommet du pergélisol, le déclenchement de phénomènes thermokarstiques catastrophiques. Le réchauffement hivernal prévu aura des conséquences plus limitées, la fusion du pergélisol ne se produisant qu'à la limite sud de celui-ci, dans la zone du pergélisol discontinu.

Sans entrer ici dans le problème très complexe des prévisions climatiques, observons que de 1947 à 1986, une partie importante de l'Arctique (comprenant le Labrador, l'île de Baffin, le Groenland, une partie de l'Océan Arctique, les mers de Kara et de Barents et toute la Scandinavie) a vu s'abaisser la température de 0,5 à $1{ }^{\circ} \mathrm{C}$ (Jones, 1990) alors qu'un réchauffement était enregistré en Sibérie orientale, en Alaska et dans l'ouest de l'Arctique Canadien. Ces variations différentes de température sont attestées d'une part par la croissance de la glace de fente de gel à la partie supérieure de coins de glace dans le Labrador, phénomènes qui prouvent l'existence d'un refroidissement et d'autre part par les mesures de température effectuées dans les sondages profonds de l'Alaska septentrional (Lachenbruch et al., 1988). Comme dans le pergélisol, il n'y a pratiquement pas de mouvement d'eaux souterraines et que le transfert de chaleur se fait presqu'uniquement par conduction, il est relativement simple de reconnaître les changements de température qui se propagent lentement en profondeur et de connaître les variations de température de surface qui leur ont donné naissance. Bien entendu les variations de température décelées se sont produites à la base de la couche active, c'est-à-dire au sommet du pergélisol et comme nous l'avons vu précédemment, elles ne reflètent pas nécessairement un réchauffement de température de l'air. 
L'ensemble des données enregistrées dans une zone de l'Alaska grande comme 7 fois la superficie de la Belgique, démontre une augmentation de température de quelques degrés pendant le $20^{\mathrm{c}}$ siècle (Nelson et al., 1993). Quelques sondages montrent un refroidissement plus récent, mais qui est interprété comme le résultat de perturbations anthropiques (Lachenbruch and Marshall, 1986).

Cette évolution différente de la température dans l'Arctique, rend plus difficiles encore la reconnaissance du début de réchauffement climatique annoncé. À notre connaissance, aucun auteur n'a encore fait savoir que des observations permettaient de reconnaître avec certitude le réchauffement. De même aucun auteur n'a, à ce jour, annoncé le développement des phénomènes thermokarstiques qui indiqueraient une élévation effective de la température.

Comme nous l'avons expliqué précédemment, la prédiction est d'autant plus difficile que, non seulement, la température, mais aussi les précipitations vont varier. C'est-à-dire que la couverture neigeuse sera sans doute modifiée, et en épaisseur, et dans sa distribution temporelle. La prévision de ce qui se passera à ce point de vue est délicate car non seulement le rôle d'isolant thermique de la neige est déterminant, mais en outre la variation de la couverture neigeuse, interviendra par l'intermédiaire de l'albedo.

L'influence des modifications de la couverture végétale induite par le réchauffement climatique vient encore compliquer les prévisions. Dans quelle mesure le développement de la végétation limitera-t-il le réchauffement du sol ? Quelles seront d'autre part les conséquences sur l'humidité du sol, d'une évaporation plus intense et d'un partiel assèchement de la couche active à la suite des modifications du drainage du sol résultant de l'abaissement du pergélisol ? La quantité d'eau qui gèle et qui dégèle dans la couche active a en effet une influence déterminante sur l'épaisseur de celle-ci et les modifications apportées par le réchauffement sur le cycle hydrologique restent très mal perçues.

La communauté des chercheurs concernés par le pergélisol ont pris conscience de l'ignorance où elle se trouve et des programmes de recherche ont été mis sur pied pour pouvoir établir des modèles de variations spatiales et temporelles de l'épaisseur de la couche active qui pourraient être couplés aux modèles généraux de variation climatique. C'est ainsi qu'un programme est en cours dans le nord de l'Alaska pour suivre les variations d'épaisseur de la couche active et les caractéristiques du sol selon une grille de $1 \mathrm{~km}$ de côté pour deux transects allant de la mer vers l'intérieur des terres et partant de Point Barrow et de Prudhoe Bay (Brown, 1994). Des programmes de recherche semblables sont en cours également au Canada et en Russie. 


\section{F. Remarques finales}

Devant de telles incertitudes, des indications sur ce qui pourrait se produire, sont recherchées par l'étude de ce qui est arrivé pendant les périodes plus chaudes qui ont existé au cours de l'Holocène et au cours du dernier interglaciaire. Les observations disponibles attestent que les modifications de température ont été particulièrement importantes dans l'arctique, mais en raison notamment de la rareté des observations, la reconstitution des conditions qui ont existé est difficile. De telles reconstructions ont été tentées en Chine (Wang, 1989) et en Russie (Brown, 1994 ; Velichko and Nechaev, 1992) principalement par des datations de traces de pergélisol ancien, mais évidemment les résultats restent très limités.

Le réchauffement climatique par ailleurs pourrait être amplifié par le dégagement de dioxyde de carbone et de méthane qui seraient libérés à la suite du réchauffement climatique dans les régions ou s'étend de nos jours un pergélisol. L'accroissement d'épaisseur de la couche active et la disparition du pergélisol pourraient accélérer la décomposition de la matière organique présente dans le sol. La libération d'une partie des 50 à $450 \mathrm{Gt}$ de carbone présents dans le sol de l'Arctique et des écosystèmes nordiques pourraient accroître l'augmentation du dioxyde de carbone dans l'atmosphère (Marion and Oechel, 1993).

Toutefois, le danger dû à cette libération possible de dioxyde de carbone est sans commune mesure avec le danger souvent évoqué de la libération de grande quantités de méthane stockées dans des cristaux de gaz hydrates ou clathrates. Les sédiments du pergélisol peuvent en effet contenir ces structures cristallines contenant de l'eau et de grandes quantités de gaz naturels (principalement du méthane). Ces clathrates sont stables sous des conditions de basse température et de pression qui, par exemple, sont rencontrées entre 200 et $700 \mathrm{~m}$ de profondeur à Barrow en Alaska. Les études récentes montrent que même dans le cas d'un réchauffement important de l'ordre de $10^{\circ} \mathrm{C}$ (qui est le changement qui survient quand la mer par érosion côtière ou à la suite d'une transgression marine s'avance sur les terres), il faudra plusieurs millénaires avant que le réchauffement n'atteigne la profondeur où il provoquera la libération de ces gaz (Nelson et al., 1993)

Au total, dans ce problème complexe des conséquences du réchauffement climatique sur le pergélisol, il manque surtout des observatións de terrain fondamentales sur la répartition détaillée du pergélisol, sur la variation spatiale des caractéristiques de la couche active, et sur la transmission du réchauffement de l'air au sommet du pergélisol. Ces 
observations sont indispensables pour prévoir valablement les effets à court terme du "global change » et déterminer où on peut en attendre les principales conséquences. À long terme et pour un réchauffement important, la disparition du pergélisol sur des étendues considérables est inéluctable avec l'apparition de phénomènes thermokarstiques de grande ampleur qui bouleverseront de grandes parties des régions arctiques.

\section{BIBLIOGRAPHIE}

Berger, A., 1992. Le climat de la terre. Un passé pour quel avenir? DeBoeck Université, $479 \mathrm{p}$.

Beskow, G.,1935. Tjälbildningen och Tjällyftningen. Statems Väfinstitut. Meddelalnde 48, Sveriges Geologiska Undersökning. Sor.C., n 375, Stockolm, 242 p.

Brown, J., 1994. Permafrost and climate change: the I.P.A. report to the $I P C C$. Frozen Ground. The new Bulletin of the international Permafrost Association, $\mathrm{n}^{\circ} 15$, p. 16-26.

Brown, R.J.E., 1970. Permafrost in Canada. University Toronto Press, 234 p.

Bunge, A. V., 1902. Einige Worte zur Bodeneisfrage. Russich-Kaiserlichin Mineral, Gesell. St. Petersburg Verh, $2^{\text {d }}$ Ser, Vol. 40 (1), pp. 203-209.

CASS, L. A. and Miller, R. D., 1959. Role of the electric double layer in the mechanism of frost heaving. - U.S. Army Snow Ice and Permafrost Research Establishment, Research report, 49, 15 p.

ChALmERS, B. and JACKSON, K. A., 1970. Experimental and theoretical studies of the mechanism of frost heaving: U.S. Army corps of Engineers, Cold Regions. Research and Engineering Laboratory Research - Report 199, $22 \mathrm{p}$.

Hansen, J., Fung, I., Lacis, A., Rind, D., LebedefF, S., Ruedy, R., et RusSEL, G., 1988. Global Climate changes as Forecast by Goddard Institute for Space Studies. Three Dimentional Model. J. Geoph. Res, 93 (D8), pp. 9341 9364.

HARRIS, S., 1986. The permafrost environment. Groom Helm London and Sydney, $276 \mathrm{p}$.

Harris, S. A., French, H. M., Heginbottom, J. A., Johnston, G. H., LadaNYI, B., SEgo, D. C., VAN Everdingen, R. D., 1988. La terminologie du pergélisol et notions connexes - Conseil national de recherches du Canada, note de service technique $\mathrm{n}^{\circ} 142,154 \mathrm{p}$.

HeginbotTom, J. A., 1993. Generalised distribution of permafrost in the northern hemisphere. Proceedings of the sixth international conference on Permafrost, Beijing China, July 05.09.1993, South China University of Technology Press, Vol. 2, p. IV.

Holmes, G. W., Hopkins, D. M. and Foster, H. L., 1968. Pingos in central Alaska - Geological Survey, Bulletin 1241, Washington U.S.A., 40 p.

Hughes, O. L., 1969. Distribution of open system pingos in Central Yukon territory with respect to glacial limits. Paper 69-34 Geological Survey of Canada, Ottawa, 8 p. 
Jonis, P. D., 1990. Le climat des mille dernières années. La Recherche $\mathrm{n}^{\circ} 219$, pp. 304-312.

JUDGE, A. S., 1973. The thermal regime of the Mackenzie valley : observations of the Natural State Environmental - Social Committee. Northern Pipelines Tasks force on Northern Oil Development. Report $n^{\circ} 73038$, Information Canada, R 72 - 11973, 177 p.

LACHENBRLCH, A. H., 1966. Contraction theory of ice wedge polygons: a qualitative discussion. Permafrost international conference (Lafayette, Indiana, 11-15 november 1963) Proceedings. Nat. Acad. Sci. Nat. Research Council, pp. 63-71.

Lachenbruch, A. H., and Marshall, B. V., 1986. Changing climate: geothermal evidence from permafrost in the Alaskan arctic. Science, 234, pp. 689-696.

Lachenbruch, A. H., Cladouhos, T.T. and Saltus, R. W., 1988. Permafrost temperature and the changing climate; Proceedings of the Fifth International Conference on Permafrost, Vol. 3, Tapir Publishers Trondheim, Norway. pp. 9-17.

LEFFINGWELL, E., 1915. Ground-ice Wedges; the dominant form of ground-ice on the north coast of Alaska. Journal of Geology, Vol. 23, pp. 635-654.

Liestol, O., 1977. Pingos, springs and permafiost in Spitsbergen. Norsk Polarinstitutt Arbok, 1975, pp. 7-29.

MaCkAY, J. R, 1972. The world of underground ice. Assoc. Am. Geographers Annals, Vol. 62, pp. 1-22.

MaCKaY, J. R., 1977. Pulsating pingos, Tuktoyaktuk peninsula, N.W.T. Can, Journ. Earth Sci, Vol. 14 (2), pp. 209-222.

MaCkaY, J. R., 1979. Pingos of the Tuktoyaktuk peninsula area, N.W.T. Geogr. Phys. et Quat. , Vol. 33 (1), pp. 3-61.

Marion, G. M. and Olchel, W. C., 1993. Mid- to Late-Holocene carbon balance in Arctic Alaska and its implications for future global warming. The Holocene, Vol. 3, pp. 193-200.

Nei.son, F. E., Lachenbruch, A. H., Woo, M. K., Koster, E. A., OsterKamp, T. E., Gavrilova, M. K., Cheng Glodong, 1993. Permafrost and changing climate. Permafrost Sixth International Conference. Proceedings (Vol. 2), July 05.09.1993, Beijing China, pp. 987-1005.

Pissart, A. et FreNCH, H. M., 1976. Pingos investigations, north-central Banks Island. Canadian Arctic. Journal canadien Sc. Terre, Vol. 13 (7), pp. 937-946.

Pissart, A., 1967. Les pingos de l'lle Prince Patrick $\left(76^{\circ} \mathrm{N}, 120^{\prime} \mathrm{W}\right.$ ). Géograph. Bull., Vol. 9, pp. 189-217.

PisSART, A., 1975. Glace de ségrégation, soulèvement du sol et phénomènes thermokarstiques dans les régions à pergélisol. Bull. Soc. Géogr. Liège, Vol. 11, pp. 89-96.

PISSART, A., 1987. Géomorphologie périglaciaire. Texte des leçons de la Chaire Franqui belge 1986. Publication du laboratoire de Géomorphologie et de Géologie du Quaternaire de l'Université de Liège, $135 \mathrm{p}$.

PORSILD, D. E.. 1938. Earth mounds in unglaciated arctic northwestern America. Geogr. Rev, Vul. 28, pp. 46-58.

Shumsky, P. A.. 1959. Principles of geocryology (permafiost studies) - Part I general geocryology, chapter IX. Ground ice - Academy of Sciences of U.S.S.R., V.A. ObRUTChEv. Institute of Permafrost Studies, Moscow, - 
pp. 274-327. Nat. Res. Conseil, technical translation 1130, translated by G. DE LEUCHTENBERG, Ottawa, 1964.

STAGER, J. K., 1956. Progress report on the analysis of the characteristics and distribution of pingos east of Mackenzie Delta. Canadian Geographer, Vol. 7, pp. 13-20.

TABER, S., 1929. Frost heaving. Journal of geology, Vol. 37 (5), pp. 428-461. VelichKo, A.A. and NechaEv, V.P., 1992. The estimation of permafrost dynamics in northern Eurasia within the global warming. Proceedings of the Academy of Sciences, Vol. 324, pp. 667-671.

Vyalov, S.S., Gerasimov, A. S., Zolotar, A. J., Fotiev, S. M., 1993. Ensuring structural stability and durability in permafrost ground areas at global warming of the earth's climate. Permafrost sixth International Conference Proceedings (Vol. 1), July 05.09.1993, Beijing China, pp. 955-960.

WANG, S., 1989. Formation and Evolution of Permafrost on the Qinghai-Xiang Plateau since the late Pleistocene. Journal of glaciology and Geocryology, Vol. II, pp. 75-81 (en chinois avec un résumé en anglais).

WILliams, P. J., 1982. The surface of the earth : an introduction to geotechnical science. Longman, London and New York, $210 \mathrm{p}$. 